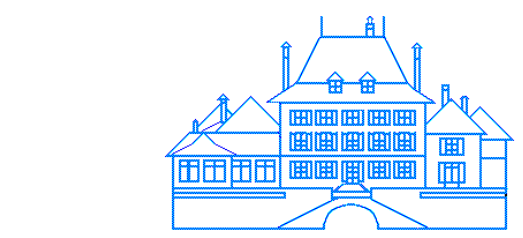

STUDIENZENTRUM GERZENSEE

STIFTUNG DER SCHWEIZERISCHEN NATIONALBANK

\title{
Higher Order Expectations in Asset Pricing
}

\section{Philippe Bacchetta and Eric van Wincoop}

\author{
Working Paper 04.03
}

This discussion paper series represents research work-in-progress and is distributed with the intention to foster discussion. The views herein solely represent those of the authors. No research paper in this series implies agreement by the Study Center Gerzensee and the Swiss National Bank, nor does it imply the policy views, nor potential policy of those institutions. 


\section{Higher Order Expectations in Asset Pricing ${ }^{1}$}

\author{
Philippe Bacchetta \\ Study Center Gerzensee \\ University of Lausanne \\ CEPR
}

\author{
Eric van Wincoop \\ University of Virginia \\ NBER
}

May 6, 2004

\footnotetext{
${ }^{1}$ We are grateful to Bernard Dumas, Hyun Shin and Narayana Kocherlakota for comments on a previous draft. Financial support by the National Centre of Competence in Research "Financial Valuation and Risk Management" (NCCR FINRISK) is gratefully acknowledged. The NCCR FINRISK is a research program supported by the Swiss National Science Foundation.
} 


\begin{abstract}
In this paper, we examine formally Keynes' idea that higher order beliefs can drive a wedge between an asset price and its fundamental value based on expected future payoffs. In a dynamic noisy rational expectations model, higher order expectations add an additional term, which we call the higher order wedge, to a standard asset pricing equation. Consistent with Keynes' reasoning we show that investment decisions are based not just on expected future payoffs, but also on anticipated future expectational errors made by the market. The latter are captured by the higher order wedge. We show that the expectation of future expectational errors by the market is perfectly rational when investors have both noisy public and private information. The main effect of this additional asset pricing term is to disconnect the price from the present value of future payoffs. We show that this effect can be quantitatively significant.
\end{abstract}




\section{Introduction}

In his General Theory, Keynes (1936) devotes significant attention to factors that can drive a wedge between an asset price and its fundamental value based on expected future payoffs. ${ }^{1}$ He emphasizes in particular two factors, mass psychology and higher order opinions. Although market psychology had largely been neglected for decades, it is now receiving significant attention in the growing field of behavioral finance. ${ }^{2}$ On the other hand, the impact of higher order expectations on the equilibrium asset price has received little attention and is not well understood.

Higher order expectations refer to expectations that investors form of other investors' expectations of an asset's subsequent payoffs. They should naturally play an important role in asset markets where investors have heterogenous information. In the words of Keynes, investors "are concerned, not with what an investment is really worth to a man who buys it for keeps, but with what the market will value it at ... three months or a year hence". In a well-known paragraph he compares asset markets to a beauty contest, where contestants have to pick the faces that other competitors find the most beautiful. Keynes argues that third and higher order expectations matter as well: "We have reached the third degree where we devote our intelligences to anticipating what average opinion expects the average opinion to be. And there are some, I believe, who practice the fourth, fifth and higher degrees."

In this paper, we formalize Keynes' ideas on the role of higher order expectations and investigate the implications for the equilibrium asset price. As anticipated by Keynes, we show that higher order expectations create a wedge between the equilibrium price and the expected value of future payoffs. More precisely, in standard asset pricing models the price depends on expected payoffs and discount rates. We show that a third determinant is added that depends on the difference between higher order and first order expectations of future payoffs and risk premia. The main objective of the paper is to understand what determines this wedge created by higher order expectations and what its implications are for the equilibrium asset price.

Our approach towards analyzing the "higher order wedge" is twofold. We first

\footnotetext{
${ }^{1}$ See Chapter 12, section 5.

${ }^{2}$ See Barberis and Thaler (2003) and Hirshleifer (2001) for surveys of the field.
} 
analyze what determines the higher order wedge in a dynamic asset pricing model with a general information and payoff structure. We also consider the implications of the wedge for the equilibrium price in this general context. Second, we examine two models with specific information and payoff structures, using the insights from the general analysis. The first model is of a finite-lived asset with one terminal payoff, which is widely studied in finance. The second model is of an infinitelylived asset with continuous payoffs and a constant information flow, leading to a time-invariant equilibrium price function. These two models are also used to show that higher order expectations can have significant quantitative implications for the equilibrium price.

A basic feature of the higher order wedge is that it depends on expectational errors of future payoffs that the average investor expects the market to make next period. This is in line with Keynes' reasoning discussed above. If investors expect that the market will value the asset too high next period, they will buy the asset, pushing up its price. This is captured by the higher order wedge, which is positive when investors systematically expect other investors to value the asset too high in future periods.

We show that it is perfectly rational for investors to expect that next period's market expectation of future payoffs is too high or too low. Investors expect the market to make expectational errors to the extent that they expect average private signals to differ from their own. Public information plays a key role here. When an investor receives private information that is less favorable than public information, he concludes that his private signal is weak and expects others to have more favorable private information. Consequently, when the average private signal is weaker than public information, the majority of investors expect others to have more favorable private signals than their own. If private signals today are still informative tomorrow, the majority of investors then expect the outlook of the market to be too favorable tomorrow. Investors buy the asset in anticipation of this, pushing up the price. Thus, it is the combination of both noisy public and private information that makes it rational for investors to expect the market to make expectational errors.

We find that one of the key implications of the higher order wedge is that it disconnects the price from the present value of future payoffs. Overly favorable public signals generally lead to both an overestimation of the present value of future 
payoffs and an overestimate of average private signals about those future payoffs. The latter leads to a positive higher order wedge, which therefore amplifies the impact on the asset price of expectational errors about future payoffs. By giving more weight to expectational errors the higher order wedge reduces the correlation between the equilibrium price and future payoffs.

Although the impact of higher order expectations has not been explicitly analyzed yet in a dynamic asset pricing model, there is a significant literature dealing with higher order expectations. We review some of this literature in the next section and argue that there has been some confusion about the role of higher order expectations. We also make clear how our paper relates to the literature. What is noteworthy about models with higher order expectations is that they can be solved without making these expectations explicit. However, this does not mean that these expectations do not matter for the equilibrium price. It is the purpose of this paper to analyze them explicitly and to show in what way they matter.

The remainder of the paper proceeds as follows. In section 3 we develop a simple asset price equation that relates the price to first and higher order expectations of future dividends and risk premia. We show that the equilibrium price is driven by three factors: expected payoffs, current and expected future risk premia and the difference between higher order and first order expectations of future payoffs and risk premia. In section 4 we analyze the determinants of the higher order wedge. We first show that it can be written in the form of expectations of future expectational errors. We then show that expectations of future expectational errors themselves depend on expectational errors about the mean set of private signals. Finally, we show that these expectational errors result from noisy public information about the mean set of private signals. In section 5 we explain why the wedge weakens the correlation between the asset price and future payoffs. This is made more precise in sections 6 and 7 , which adopt specific payoff and information structures. In section 6 we consider a finitely-lived asset with only one payoff, while in section 7 we study an infinitely-lived asset with continuous payoffs. Section 8 concludes. 


\section{Related Literature}

While higher-order beliefs have been studied in a wide range of contexts, two features make them of special interest in the context of financial markets. First, in financial markets the price today depends on the price tomorrow, so that investors naturally need to form expectations of future market expectations. This dynamic perspective differs from the analysis of 'static' higher order expectations, i.e., expectations of expectations within a period. This is the case when agents interact strategically, e.g., as in Morris and Shin (2002), Woodford (2003) or Amato and Shin (2003). ${ }^{3}$ We abstract from strategic interactions by assuming atomistic investors. Second, in financial markets the price provides a mechanism through which idiosyncratic information is aggregated. In forming expectations of other investors' expectations, special attention is paid to the asset price as it is informative about the private information of others. This additional feature is often not present in the analysis of games with incomplete information, e.g., in global games.

We will show that equilibrium asset prices depend on a dynamic form of higher order expectations. It depends on the average expectation at time $t$ of the dividend at $t+1$, the average expectation at $t$ of the average expectation at $t+1$ of the dividend at $t+2$, and so on. This is the first paper to explicitly solve for the equilibrium price as a function of higher order expectations of all future payoffs. It allows us to explicitly analyze the implications of higher order expectations for the equilibrium price. While dynamic asset price models with higher order expectations have been studied in several papers, the implications of the models are generally analyzed without any reference to higher order expectations. This can be done because these models can be solved using a reduced form where higher order expectations are not explicit. This was first shown by Townsend (1983) in the context of a dynamic business cycle model that features dynamic higher order expectations. ${ }^{4}$

\footnotetext{
${ }^{3}$ Hellwig (2003) characterizes explicitly higher order expectations in the model proposed by Woodford (2003). It is therefore related in spirit to our approach.

${ }^{4}$ The general approach is the method of undetermined coefficients. In the context of asset pricing one first assumes some equilibrium asset price as a linear function of current and past innovations. Investors make decisions based on this conjectured price equation. The resulting equilibrium price equation is then equated to the conjectured one in order to solve for the
} 
Most of the literature on asset pricing and higher order expectations considers a special model where an asset has only one payoff at a terminal date. ${ }^{5}$ Investors receive private information on the final payoff either at an initial date or every period. They trade every period and progressively learn about the final payoff by observing the price. Such a model is studied in particular by He and Wang (1995), Vives (1995), Foster and Viswanathan (1996), Brennan and Cao (1997), and Allen, Morris, and Shin (2003). ${ }^{6}$ Several interesting insights have been drawn by previous authors, ${ }^{7}$ but only Allen et. al. (2003) explicitly analyze the role of higher order expectations for the equilibrium price.

He and Wang (1995) and Foster and Viswanathan (1996) show that higher order expectations can be reduced to first order expectations. However, the higher order expectations that they refer to do not influence the equilibrium asset price. They focus on static higher order expectations (the average expectation at time $t$ of the average expectation at time $t$ ) instead of the dynamic higher order expectations discussed above that the equilibrium price depends on. Even though these authors do not consider the relevant higher order expectations, they are correct in arguing that higher order expectations can be reduced to first order expectations. In this paper we show that the higher order wedge depends on the average first order expectational error about the mean set of private signals. It is important to stress though that the ability to reduce higher order to first order expectations does not imply that they do not matter. The wedge created by higher order expectations is an additional determinant of the asset price, separate from expected dividends and risk premia, and can be quantitatively very large. ${ }^{8}$

coefficients.

${ }^{5}$ See Brunnermeier (2001) for a nice survey of the literature. There are a few papers that work with somewhat alternative models, e.g., Biais and Bossaerts (1998).

${ }^{6}$ Foster and Viswanathan (1996) consider a model with strategic trading, while the other papers consider competitive investors.

${ }^{7}$ Among the issues analyzed are trading volume and intensity, market depth and liquidity, the informativeness of prices, as well as important aspects of the solution procedure.

${ }^{8} \mathrm{He}$ and Wang (1995) and Foster and Viswanathan (1996) argue that the ability to reduce higher order to first order expectations helps solve the model since the infinite space of mean beliefs that Townsend (1983) alluded to is reduced to a space of only first order beliefs. However, the method of undetermined coefficients used by Townsend to solve the model does not make any reference to the space of mean beliefs and the solution methods in these two papers also make no use of the fact that higher order expectations can be reduced to first order expectations. 
Allen et. al. (2003) focus more closely on the role of higher order expectations in the terminal payoff model. They do not analyze the higher order wedge, but compare the equilibrium price function over time. The farther we are from the terminal date, the higher the order of expectations. They find that more weight is given to public information the higher the order of expectations. A limitation of that approach is that the equilibrium price function can also change over time due to an expanding information set. In this paper we focus on the higher order wedge since it allows us to more precisely identify the role of higher order expectations. We also develop the role of noisy public information in a more general environment. We show that it leads to expectational errors about the mean set of private signals, which contributes to disconnecting the price from future payoffs.

While the terminal payoff model is technically convenient, it is not very realistic. A few papers have analyzed asset pricing models with higher order expectations in a more realistic dynamic environment with an infinitely-lived asset yielding dividends each period and with a constant flow of information. Such models lead to time-independent second order moments and are more in the tradition of stochastic dynamic macroeconomic models. Higher order expectations in an infinite horizon framework were indeed first analyzed in macroeconomics, in the business cycle model of Townsend (1983). The first paper in finance to analyze such a model is Singleton (1987). He focuses on the time series properties of the equilibrium price, without considering the role of higher order expectations. In Bacchetta and van Wincoop (2003), we solve an infinite horizon model of exchange rate determination in which higher order expectations arise. Using the results from the present paper, we show that higher order expectations can help contribute to the puzzling disconnect between the exchange rate and observed macroeconomic aggregates.

\section{A Simple Asset Pricing Equation}

In this section we derive a simple asset price equation that relates the asset price to higher order expectations of future payoffs. We adopt a share economy that is standard in the noisy rational expectations literature and allows for an exact solution without using linearization methods. The basic assumptions are: i) constant absolute risk aversion; ii) a normally distributed excess return; iii) a constant 
risk-free interest rate; iv) a share economy with a stochastic supply of shares; v) investors invest for one period only (overlapping-generations of two-period lived investors); vi) a competitive market with a continuum of investors on the interval $[0,1]$.

Investors allocate optimally their wealth between a risky stock and a safe asset. Let $P_{t}$ be the ex-dividend share price, $D_{t}$ the dividend, and $R$ the constant gross interest rate. The dollar excess return on one share is $Q_{t+1}=P_{t+1}+D_{t+1}-R P_{t}$. This leads to the standard asset demand equation

$$
x_{t}^{i}=\frac{E_{t}^{i}\left(P_{t+1}+D_{t+1}\right)-R P_{t}}{\gamma \sigma_{t}^{2}}
$$

where $\gamma$ is the rate of absolute risk aversion and $\sigma_{t}^{2}$ is the conditional variance of next period's excess return.

The per capita random supply of shares is $X_{t}$, so that the equilibrium price $P_{t}$ follows from the market clearing condition $\int_{0}^{1} x_{t}^{i} d i=X_{t}$. As usual, we assume that average random variables are equal to their expected values ${ }^{9}$ and we define the average or market expectation as $\bar{E}_{t}=\int_{0}^{1} E_{t}^{i} d i$. If we define the risk premium term as $\phi_{t}=\gamma \sigma_{t}^{2} X_{t} / R$, the market clearing condition gives:

$$
P_{t}=\frac{1}{R} \bar{E}_{t}\left(P_{t+1}+D_{t+1}\right)-\phi_{t} .
$$

To compute the equilibrium price, we need to integrate (2) forward. In typical asset pricing formulas, this is done by applying the law of iterated expectations. While this law always holds for individual expectations, it may not hold for market expectations when investors have different information sets. For example, $\bar{E}_{t} \bar{E}_{t+1} D_{t+2} \neq \bar{E}_{t} D_{t+2} \cdot{ }^{10}$ Thus, we define the average expectation of order $k$ as

$$
\bar{E}_{t}^{k}=\bar{E}_{t} \bar{E}_{t+1} \ldots \bar{E}_{t+k-1}
$$

for $k>1$. Moreover, $\bar{E}_{t}^{0} x=x, \bar{E}_{t}^{1} x=\bar{E}_{t} x$. The equilibrium price is then (ruling out bubbles):

$$
P_{t}=\sum_{s=1}^{\infty} \frac{1}{R^{s}} \bar{E}_{t}^{s} D_{t+s}-\sum_{s=1}^{\infty} \frac{1}{R^{s}} \bar{E}_{t}^{s} \phi_{t+s}-\phi_{t}
$$

\footnotetext{
${ }^{9}$ See Admati (1985) for a discussion.

${ }^{10}$ See also Allen, Morris, and Shin (2003) for a nice illustration.
} 
The stock price is equal to the present discounted value of expected dividends minus risk premia. The difference with a standard asset pricing equation is that first order expectations are replaced by higher order expectations. A dividend accruing $s$ periods ahead has an expectation of order $s$. For example, if $s=2$, we need to compute the market expectation at time $t$ of the market expectation at $t+1$ of $D_{t+2}$ rather than the first-order expectation of $D_{t+2}$. This implies that investors have to predict the future market expectation of the dividend rather than the dividend itself. This is the 'beauty contest' phenomenon described by Keynes. Moreover, with an infinite horizon, the order of expectation can obviously go to infinity.

To examine the impact of higher order expectations on asset prices, we look at differences between first and higher order expectations. This higher order wedge is defined as

$$
\Delta_{t}=\sum_{s=2}^{\infty} \frac{1}{R^{s}}\left[\bar{E}_{t}^{s} D_{t+s}-\bar{E}_{t} D_{t+s}\right]-\sum_{s=2}^{\infty} \frac{1}{R^{s}}\left[\bar{E}_{t}^{s} \phi_{t+s}-\bar{E}_{t} \phi_{t+s}\right]
$$

It depends on the present value of deviations between higher order and first order expectations of dividends minus risk premia. The higher order wedge $\Delta_{t}$ adds a third element to the standard asset pricing equation:

$$
P_{t}=\sum_{s=1}^{\infty} \frac{1}{R^{s}} \bar{E}_{t} D_{t+s}-\left(\sum_{s=1}^{\infty} \frac{1}{R^{s}} \bar{E}_{t} \phi_{t+s}+\phi_{t}\right)+\Delta_{t}
$$

The first term is associated with expected payoffs; the second term captures current and expected future risk premia (affecting discount rates); the last term is the higher order wedge.

We will define the present value of dividends minus risk premia at $t$ as $P V_{t}=$ $\sum_{s=1}^{\infty} \frac{1}{R^{s}}\left(D_{t+s}-\phi_{t+s}\right)$, which allows us to also write the asset price as

$$
P_{t}=\bar{E}_{t} P V_{t}-\phi_{t}+\Delta_{t}
$$

which will be used below.

\section{The Higher Order Wedge}

In this section, we examine the determinants of the additional term in the asset pricing equation under a general information structure. First, we show that the 
higher order wedge is determined by first and higher order expectations of future expectational errors. For example, investors at time $t$ need to estimate the market expectation at $t+1$ of the market estimation mistake at $t+2$ about the present value of dividends. Second, we show how the expectation of future expectational errors, and thus the wedge, is proportional to the first order expectational error about average private signals. Third, we examine the conditions under which the wedge exists and under which it is largest. We show that the presence of public information plays a crucial role and that the wedge is largest for intermediate degrees of the quality of private information.

For expositional purposes only, we will assume that the second term in (5) is zero, so that the higher order wedge is only associated with the difference between higher and first order expectations of dividends. This will for example be the case when there is only public information about future risk premia. We want to emphasize though that the results in this section are general. Everything we say below holds for the general case where higher order expectations of both future dividends and risk premia differ from first order expectations. One simply needs to replace the word "dividend" by "dividend minus risk-premium" in what follows.

\subsection{Expectations of Future Expectational Errors}

We first show that the difference between higher and first order expectations in (5) can be written in terms of expectations of market expectational errors. This has two advantages. First, it makes concrete the conjecture by Keynes (1936) that investors do not just make decisions based on their own perception of the "prospective yield" (expected future dividends), but worry about market expectations. Second, it allows us to adopt an iterative procedure in section 4.3 to convert the wedge into an expression that depends on first order expectational errors about average private signals.

First consider $s=2$. The difference between the second and first order expectation is equal to the average expectation at time $t$ of the average expectational error at $t+1$ about $D_{t+2}$ :

$$
\bar{E}_{t}^{2} D_{t+2}-\bar{E}_{t} D_{t+2}=\bar{E}_{t}\left(\bar{E}_{t+1} D_{t+2}-D_{t+2}\right)
$$

The intuition behind this term is as follows. Investment decisions at time $t$ are based on the expected price at $t+1$. This price will reflect the market expectation of 
subsequent dividends. An investor at time $t$ therefore makes investment decisions not just based on what he believes the dividend at $t+2$ to be, but also on whether he believes the market to make an expectational error at $t+1$ about the dividend at $t+2$. When investors have common information, they expect no future market expectational errors. But as we show below, this is no longer the case when information is heterogeneous.

Next consider $s=3$. The difference between the third and first order expectation is equal to the difference between the first and second order expectation plus the difference between the second and third order expectation. This can be written as the average expectation at $t$ of the average expectational error at $t+1$ plus the second order expectation at $t$ of the average expectational error at $t+2$ :

$$
\bar{E}_{t}^{3} D_{t+3}-\bar{E}_{t} D_{t+3}=\bar{E}_{t}\left(\bar{E}_{t+1} D_{t+3}-D_{t+3}\right)+\bar{E}_{t}^{2}\left(\bar{E}_{t+2} D_{t+3}-D_{t+3}\right)
$$

The last term can be understood as follows. Just as the price at time $t$ depends on expected average expectational error at $t+1$, so does the price at $t+1$ depend on expected average expectational error at $t+2$. The expected return from $t$ to $t+1$ then depends on the expectation at time $t$ of the market's expectation at $t+1$ of the market's expectational error at $t+2$. In other words, investment decisions at time $t$ depend on the second order expectation at $t$ of the market's expectational error at $t+2$.

Proceeding along this line for expectations of even higher order, we can rewrite (5) as follows: ${ }^{11}$

$$
\Delta_{t}=\sum_{s=1}^{\infty} \frac{1}{R^{s+1}} \bar{E}_{t}^{s}\left(\bar{E}_{t+s} P V_{t+s}-P V_{t+s}\right)
$$

The higher order wedge therefore depends on first and higher order expectations of future expectational errors of the subsequent present value of dividends: the market expectation at $t$ of the market's expectational error at $t+1$ of $P V_{t+1}$, the second order expectation at $t$ of the expectational error at $t+2$ of $P V_{t+2}$, and so on. Investors make decisions not just based on what they expect future dividends to be, but also on what they expect the market's expectational error next period to be about those future dividends, and what they expect next period's market

\footnotetext{
${ }^{11}$ For each $s$ rewrite the difference between the $s$-order and first order expectation as $\bar{E}_{t}^{s} D_{t+s}-$ $\bar{E}_{t} D_{t+s}=\left(\bar{E}_{t}^{2} D_{t+s}-\bar{E}_{t} D_{t+s}\right)+\left(\bar{E}_{t}^{3} D_{t+s}-\bar{E}_{t}^{2} D_{t+s}\right)+\ldots+\left(\bar{E}_{t}^{s} D_{t+s}-\bar{E}_{t}^{s-1} D_{t+s}\right)$.
} 
expectation of the expectational error in the subsequent period to be. In the rest of our analysis we will use (8) instead of (5) to interpret the wedge.

\subsection{A General Information Structure}

In order to describe what determines the expectations of future expectational errors, as expressed in (8), we need to be somewhat more precise about the information structure. Investors have both public and private information. At time $t$, investor $i$ has a vector $\mathbf{W}_{t}^{i}$ of private signals and a vector $\mathbf{Z}_{t}$ of public signals. These are signals obtained at time $t$ and earlier that remain informative at $t$ about the present value of future dividends. We assume these vectors are finite. Average private signals are denoted $\overline{\mathbf{W}}_{t}$. We make three assumptions about the information structure.

Assumption 1 The vectors $\mathbf{W}_{t}^{i}, \mathbf{Z}_{t}, \overline{\mathbf{W}}_{t}$ and $P V_{t}$ have the same joint normal distribution for all investors $i$.

In general, some elements of the vector $\mathbf{Z}_{t}$ are endogenous, but normality typically holds in linear models.

The two other assumptions relate to the timing of private signals. It is useful to consider subsets of the vector of private signals. Let $\mathbf{v}_{t}^{i}$ be the set of new signals obtained at time $t ; \mathbf{V}_{t-1}^{i}$ the set of private signals obtained at $t-1$ and earlier that remain informative at time $t$ about future dividends; and $\mathbf{w}_{t}^{i}$ the set of signals informative at $t$, that are no longer informative at $t+1$ about the present value of future dividends. A private signal is informative about the present value when it affects the expectation of the present value conditional on all other information. ${ }^{12}$ As before, the vectors $\overline{\mathbf{v}}_{t}, \overline{\mathbf{V}}_{t}$, and $\overline{\mathbf{w}}_{t}$ denote average signals. We can then break down the vector $\mathbf{W}_{t}^{i}$ of private signals in two different ways: ${ }^{13}$

$$
(1): \mathbf{W}_{t}^{i}=\left[\begin{array}{c}
\mathbf{v}_{t}^{i} \\
\mathbf{V}_{t-1}^{i}
\end{array}\right] \quad(2): \mathbf{W}_{t}^{i}=\left[\begin{array}{c}
\mathbf{V}_{t}^{i} \\
\mathbf{w}_{t}^{i}
\end{array}\right]
$$

A crucial aspect of the analysis is that investors need to estimate future market

\footnotetext{
${ }^{12} \mathrm{~A}$ signal that is informative today may no longer be informative in the future. For example, private information about a future dividend is no longer informative once that future dividend is observed.

${ }^{13}$ The elements of the vector $\mathbf{W}_{t}^{i}$ are generally ordered differently in these two breakdowns.
} 
expectations and thus information available in the future. We make two assumptions related to private information at $t+1$.

Assumption 2 Idiosyncratic errors $\mathbf{v}_{t+1}^{i}-\overline{\mathbf{v}}_{t+1}$ are orthogonal to previous information: $E_{t}^{i}\left(\mathbf{v}_{t+1}^{i}-\overline{\mathbf{v}}_{t+1} \mid \mathbf{Z}_{t}, \mathbf{W}_{t}^{i}\right)=0$.

Assumption 3 Private signals $\mathbf{w}_{t}^{i}$ that are no longer informative at $t+1$ about $P V_{t+1}$ are also no longer informative at $t+1$ about $\overline{\mathbf{V}}_{t+1}$.

Assumption 2 says that investors cannot predict idiosyncratic errors in their own future private signals. Assumption 3 says that once private information is no longer informative about future dividends it is also not informative about average private signals that predict future dividends. Thus we can write : $\operatorname{cov}\left(\mathbf{w}_{t}^{i}, \overline{\mathbf{V}}_{t+1} \mid \mathbf{Z}_{t+1}, \mathbf{W}_{t+1}^{i}\right)=$ 0 . Using these assumptions about the general information structure, we can now be more precise about what determines the higher order wedge.

\subsection{The Wedge as a First Order Expectational Error}

Assumptions 1 and 3 imply that expectations at $t+1$ about the present value of future dividends and average private signals $\overline{\mathbf{V}}_{t+1}$ are a linear function of signals $\mathbf{W}_{t+1}^{i}$ and $\mathbf{Z}_{t+1}$. This function is the same for all investors. If we break down $\mathbf{W}_{t+1}^{i}$ into $\mathbf{V}_{t}^{i}$ and $\mathbf{v}_{t+1}^{i}$, we can write

$$
\begin{aligned}
& E_{t+1}^{i} P V_{t+1}=\boldsymbol{\theta}_{t+1}^{\prime} \mathbf{V}_{t}^{i}+\boldsymbol{\beta}_{t+1}^{\prime} \mathbf{v}_{t+1}^{i}+\gamma_{t+1}^{\prime} \mathbf{Z}_{t+1} \\
& E_{t+1}^{i} \overline{\mathbf{V}}_{t+1}=\Psi_{t+1}^{\prime} \mathbf{V}_{t}^{i}+\boldsymbol{\mu}_{t+1}^{\prime} \mathbf{v}_{t+1}^{i}+\lambda_{t+1}^{\prime} \mathbf{Z}_{t+1}
\end{aligned}
$$

In the Appendix we then derive the following Proposition about the higher order wedge.

Proposition 1 Under assumptions 1 to 3, the deviation between higher and first order expectations that affects the equilibrium asset price is

$$
\Delta_{t}=\Pi_{t}^{\prime}\left(\bar{E}_{t} \overline{\mathbf{V}}_{t}-\overline{\mathbf{V}}_{t}\right)
$$

where $\boldsymbol{\Pi}_{t}=\frac{1}{R^{2}} \boldsymbol{\theta}_{t+1}+\sum_{s=2}^{\infty} \frac{1}{R^{s+1}} \boldsymbol{\Psi}_{t+1} \ldots \boldsymbol{\Psi}_{t+s-1} \boldsymbol{\theta}_{t+s}$.

The proposition tells us that the higher order wedge depends on the average expectational error at time $t$ about the vector of average private signals. The 
proposition therefore reduces differences between higher and first order expectations to a simple first order expectational error. In section 4.4 we discuss how the combination of noisy public and private information implies average expectational errors of the average private signals $\left(\bar{E}_{t} \overline{\mathbf{V}}_{t}-\overline{\mathbf{V}}_{t} \neq 0\right)$. For now we take it as given and provide intuition about how average expectational errors about average private signals affect the higher order wedge as in Proposition 1.

The vector $\boldsymbol{\Pi}_{t}$ multiplying $\bar{E}_{t} \overline{\mathbf{V}}_{t}-\overline{\mathbf{V}}_{t}$ is implied by the sum of first and higher order expectations in (8). Consider the first element of $\boldsymbol{\Pi}_{t}$, which corresponds to the average expectation at $t$ of the market expectational error at $t+1$ about $P V_{t+1}$. An investor's expectation of this error can be written as $E_{t}^{i}\left(\bar{E}_{t+1} P V_{t+1}-P V_{t+1}\right)=$ $E_{t}^{i}\left(\bar{E}_{t+1} P V_{t+1}-E_{t+1}^{i} P V_{t+1}\right)$. From (10) it follows that:

$$
\bar{E}_{t+1} P V_{t+1}-E_{t+1}^{i} P V_{t+1}=\boldsymbol{\theta}_{t+1}^{\prime}\left(\overline{\mathbf{V}}_{t}-\mathbf{V}_{t}^{i}\right)+\boldsymbol{\beta}_{t+1}^{\prime}\left(\overline{\mathbf{v}}_{t+1}-\mathbf{v}_{t+1}^{i}\right)
$$

An investor expects the market to make expectational errors to the extent that the market is expected to have a different set of private signals. It follows from Assumption 2 that the second term in (13) is expected to be zero. An investor only expects the market to make expectational errors tomorrow if he expects the average private signals $\overline{\mathbf{V}}_{t}$ today to be different from his own private signals. Taking the expectation of (13) for investor $i$ at time $t$ yields $\boldsymbol{\theta}_{t+1}^{\prime}\left(E_{t}^{i} \overline{\mathbf{V}}_{t}-\mathbf{V}_{t}^{i}\right)$. The average of this across investors is $\boldsymbol{\theta}_{t+1}^{\prime}\left(\bar{E}_{t} \overline{\mathbf{V}}_{t}-\overline{\mathbf{V}}_{t}\right)$, which corresponds to the first element of $\Pi_{t}$.

The second element in $\boldsymbol{\Pi}_{t}$ corresponds to the sum of higher order expectations of future expectational errors. Consider for example the second-order expectation of the market's expectational error at $t+2$ about $P V_{t+2}$. Corresponding to the discussion above, the average expectation at $t+1$ of the market's expectational error at $t+2$ about $P V_{t+2}$ is $\boldsymbol{\theta}_{t+2}^{\prime}\left(\bar{E}_{t+1} \overline{\mathbf{V}}_{t+1}-\overline{\mathbf{V}}_{t+1}\right)$. This depends itself on an average expectational error, this time not about future dividends but about average private signals. Using a similar argument as above, but using (11), the average expectation at time $t$ of the market's expectational error at $t+1$ about average private signals is equal to $\boldsymbol{\Psi}_{t+1}^{\prime}\left(\bar{E}_{t} \overline{\mathbf{V}}_{t}-\overline{\mathbf{V}}_{t}\right)$. Following an iterative argument one can similarly derive third and higher order expectations of future expectational errors. The critical point is that these all depend on average expectational errors at time $t$ about average private signals.

One can think of first and higher order expectations of future expectational 
errors as resulting from a chain effect. This explains why current expectational mistakes $\bar{E}_{t} \overline{\mathbf{V}}_{t}-\overline{\mathbf{V}}_{t}$ affect expectations of all orders. As an illustration consider the case where investors receive only one private signal $v_{t}^{i}$ at time $t$ that is still relevant at $t+1$. Assume that a higher private signal $v_{t}^{i}$ at time $t$ makes the investor both more optimistic at $t+1$ about future payoffs $\left(\theta_{t+1}>0\right)$ and more optimistic at $t+1$ about average private signals $\left(\boldsymbol{\Psi}_{t+1}>0\right)$.

Now consider what happens when the average investor at time $t$ expects others to have more favorable, and therefore too optimistic, private signals, i.e., $\bar{E}_{t} \bar{v}_{t}>\bar{v}_{t}$. The average investor then expects that (1) the market is too optimistic at $t+1$ about future dividends and (2) the market is too optimistic at $t+1$ about average private signals. The first leads to first order expectations of positive expectational errors at $t+1$ about $P V_{t+1}$. The second implies a first order expectation of positive expectational errors at $t+1$ about private signals, i.e., a first order expectation at $t$ that $\bar{E}_{t+1} \bar{v}_{t+1}>\bar{v}_{t+1}$. This leads to the next step in the chain. Following the same argument as above, it leads to second order expectations that the market is too optimistic at $t+2$ about future dividends and average private signals. The latter leads to a third step in the chain, and so on.

\subsection{On the Existence and Magnitude of the Higher Order Wedge}

With Proposition 1 as a starting point, we can derive the following two related Propositions.

Proposition 2 Two necessary conditions for the existence of a higher order wedge are: (i) public information is informative about average private signals, (ii) some of the private information available at time $t$ is still informative at $t+1$ in forming expectations about future dividends.

Proposition 3 The higher order wedge depends on average expectational errors about the average set of private signals based on public information, $\bar{E}_{t}\left(\overline{\mathbf{W}}_{t} \mid \mathbf{Z}_{t}\right)-$ $\overline{\mathbf{W}}_{t}$.

The first condition for the existence of the higher order wedge is that public information is used to form expectations of average private signals. Since, from 
Assumption 1, private signals of all investors are drawn from the same distribution, based on private information alone investors expect average private signals to be the same as their own: $E_{t}^{i}\left(\overline{\mathbf{W}}_{t} \mid \mathbf{W}_{t}^{i}\right)=\mathbf{W}_{t}^{i}$. Averaging across investors it follows that $\bar{E}_{t} \overline{\mathbf{W}}_{t}=\overline{\mathbf{W}}_{t}$, and therefore also $\bar{E}_{t} \overline{\mathbf{V}}_{t}=\overline{\mathbf{V}}_{t}$, when these expectations are conditioned on private information only.

This is no longer the case once we introduce public information. When investors have public information, the expectation of average private signals generally differs from their own private signals, so that $E_{t}^{i} \overline{\mathbf{V}}_{t} \neq \mathbf{V}_{t}^{i}$. For example, assume that public information $\mathbf{Z}_{t}$ is on average more favorable about $\overline{\mathbf{V}}_{t}$ than the investors' own private signals. Then a majority of investors will expect others to have more favorable private information than their own, so that $\bar{E}_{t} \overline{\mathbf{V}}_{t}>\overline{\mathbf{V}}_{t}$.

More generally, $\bar{E}_{t} \overline{\mathbf{V}}_{t}-\overline{\mathbf{V}}_{t}$ depends on expectational errors about average private signals caused by public information. This can be seen as follows. From the projection theorem for normally distributed variables, it follows that

$$
E_{t}^{i} \overline{\mathbf{W}}_{t}=E_{t}\left(\overline{\mathbf{W}}_{t} \mid \mathbf{Z}_{t}\right)+\operatorname{cov}\left(\overline{\mathbf{W}}_{t}, \mathbf{W}_{t}^{i} \mid \mathbf{Z}_{t}\right)\left(\operatorname{var}\left(\mathbf{W}_{t}^{i} \mid \mathbf{Z}_{t}\right)\right)^{-1}\left(\mathbf{W}_{t}^{i}-E_{t}\left(\overline{\mathbf{W}}_{t} \mid \mathbf{Z}_{t}\right)\right)
$$

Taking the average over all investors and subtracting $\overline{\mathbf{W}}_{t}$ on each side, we have ${ }^{14}$

$$
\bar{E}_{t} \overline{\mathbf{W}}_{t}-\overline{\mathbf{W}}_{t}=\operatorname{var}\left(\mathbf{W}_{t}^{i}-\overline{\mathbf{W}}_{t} \mid \mathbf{Z}_{\mathbf{t}}\right)\left(\operatorname{var}\left(\mathbf{W}_{t}^{i} \mid \mathbf{Z}_{t}\right)\right)^{-1}\left(E_{t}\left(\overline{\mathbf{W}}_{t} \mid \mathbf{Z}_{t}\right)-\overline{\mathbf{W}}_{t}\right)
$$

Since average private signals $\overline{\mathbf{V}}_{t}$ are a subset of $\overline{\mathbf{W}}_{t}$, it follows that the average expectational error $\bar{E}_{t} \overline{\mathbf{V}}_{t}-\overline{\mathbf{V}}_{t}$ depends on $E_{t}\left(\overline{\mathbf{W}}_{t} \mid \mathbf{Z}_{t}\right)-\overline{\mathbf{W}}_{t}$, which are the average expectational errors about average private signals based on public information. This leads to Proposition 3.

The second condition for the existence of the higher order wedge in Proposition 2 is that private information is still relevant the next period in forming expectations of future dividends. Investors will generally believe at time $t$ that they have different private information than others. But when private information at time $t$ is no longer relevant at $t+1$, investors have no reason to believe that their information set at $t+1$ differs from others. Therefore they have no reason to expect that the market will make expectational errors at $t+1$. More formally, this condition implies that $\boldsymbol{\theta}_{t+1} \neq 0$ in (10). It then follows from equation (13) and the discussion

\footnotetext{
${ }^{14}$ Here we use that $\operatorname{cov}\left(\mathbf{W}_{t}^{i}-\overline{\mathbf{W}}_{t}, \overline{\mathbf{W}}_{t} \mid \mathbf{Z}_{t}\right)=0$ because private signals of all investors are drawn from the same distribution.
} 
below it that investors at time $t$ do not expect the market to make expectational errors at $t+1 .^{15}$

A fourth proposition provides insight about when the higher order expectation wedge is largest.

Proposition 4 The variance of $\Delta_{t}$ is largest for intermediate levels of the quality of private information. It vanishes when the idiosyncratic noise in private signals approaches zero or infinity.

The Appendix provides a formal proof. The idiosyncratic noise in private signals approaches zero when $\operatorname{var}\left(\mathbf{W}_{t}^{i}-\overline{\mathbf{W}}_{t}\right) \rightarrow 0$. It approaches infinity when there is a series of normal distributions indexed by $k$ such that in the limit, as $k \rightarrow \infty, \operatorname{var}\left(\mathbf{W}_{t}^{i}-\overline{\mathbf{W}}_{t}\right)$ approaches $k \overline{\boldsymbol{\Sigma}}_{t}$ with $\overline{\boldsymbol{\Sigma}}_{t}$ a matrix containing finite values.

A brief intuitive description for Proposition 4 goes as follows. Private information vanishes when either the idiosyncratic errors of private signals are infinitely noisy or when the noise approaches zero. In the first case private information becomes uninformative, while in the second case all investors receive the same signals, which are therefore no longer private. Investors know that all other investors have the same information and there is no reason to expect other investors to make expectational errors in the future.

\section{The Disconnect between Asset Prices and Fu- ture Dividends}

So far we have discussed the determinants of the higher order wedge. We now turn to the impact of the wedge on the equilibrium price. The main impact is that the higher order wedge tends to weaken the relationship between the asset price and the present value of future dividends. Although at this level of generality there is no formal proof of this result, it holds more generally than in the specific models discussed in the next two sections. We also show that the wedge reduces the asset price variance.

\footnotetext{
${ }^{15}$ Note that for a non-zero higher order wedge it is sufficient that private information at $t$ is still relevant at $t+1$. Private information at time $t$ does not need to be relevant at $t+2$ or later in forming expectations about future dividends or average private signals.
} 
Making explicit the expectational error in the expected present value of dividends, we can rewrite (7) as:

$$
P_{t}=P V_{t}+\left(\bar{E}_{t} P V_{t}-P V_{t}\right)-\phi_{t}+\Delta_{t}
$$

Even in the absence of the higher order wedge the correlation between $P_{t}$ and $P V_{t}$ is generally reduced by expectational errors $\bar{E}_{t} P V_{t}-P V_{t}$ and the risk-premium $\phi_{t}$. First, since the risk premium is generated by supply shocks, it may be uncorrelated with $P V_{t}$. Second, the average expectational error $\bar{E}_{t} P V_{t}-P V_{t}$ is negatively correlated with the present value of future dividends $P V_{t}{ }^{16}$

The higher order wedge further contributes to lower this correlation, because in general it is also negatively correlated with $P V_{t}$. One can write the covariance between the higher order wedge and $P V_{t}$ as

$$
\begin{aligned}
\operatorname{cov}\left(P V_{t}, \Delta_{t}\right)= & \int_{0}^{1} \operatorname{cov}\left(P V_{t}, \boldsymbol{\Pi}_{t}^{\prime}\left(E_{t}^{i} \overline{\mathbf{V}}_{t}-\overline{\mathbf{V}}_{t}\right)\right) d i= \\
& -\int_{0}^{1} \operatorname{cov}\left(E_{t}^{i} P V_{t}-P V_{t}, \mathbf{\Pi}_{t}^{\prime}\left(E_{t}^{i} \overline{\mathbf{V}}_{t}-\overline{\mathbf{V}}_{t}\right)\right) d i
\end{aligned}
$$

While the wedge depends on expectational errors about average private signals, these tend to be positively related to expectational errors about future dividends. For example, favorable public information that causes investors to be too optimistic about future dividends generally also causes them to be too optimistic about average private signals of those future dividends. Therefore $\operatorname{cov}\left(P V_{t}, \Delta_{t}\right)<0$ to the extent that expectational errors about future dividends are positively related to expectational errors about average private signals. ${ }^{17}$ The higher order wedge therefore further weakens the relationship between the price and future dividends.

In the simple model of the next section average private signals $\overline{\mathbf{V}}_{t}$ are equal to a terminal dividend $D_{T}$. With a zero interest rate, $P V_{t}$ is also equal to $D_{T}$. Therefore $\overline{\mathbf{V}}_{t}=P V_{t}$. In that case, expectational errors about $P V_{t}$ and $\overline{\mathbf{V}}_{t}$ are perfectly correlated and the higher order wedge amplifies the impact on the asset price of expectational errors about the future dividend.

\footnotetext{
${ }^{16} \operatorname{cov}\left(\bar{E}_{t} P V_{t}-P V_{t}, P V_{t}\right)=\int_{0}^{1} \operatorname{cov}\left(E_{t}^{i} P V_{t}-P V_{t}, P V_{t}\right) d i=\int_{0}^{1} \operatorname{cov}\left(E_{t}^{i} P V_{t}-P V_{t}, P V_{t}-\right.$ $\left.E_{t}^{i} P V_{t}\right) d i=-\int_{0}^{1} \operatorname{var}\left(E_{t}^{i} P V_{t}-P V_{t}\right) d i<0$.

${ }^{17}$ This implicitly assumes that the elements of $\boldsymbol{\Pi}_{t}$ are positive. Proposition 1 shows that this is naturally the case. The elements of $\boldsymbol{\Pi}_{t}$ are positive to the extent that higher private signals raise the expectation of $P V_{t}(\theta>0)$ and raise the expectation of average private signals next period $(\Psi>0)$. In the models of section 6 and 7 the vector $\Pi_{t}$ is always positive.
} 
The finding that higher order expectations disconnect the price from future dividends does not mean that they lead to more asset price volatility. On the contrary, the higher order wedge reduces asset price volatility. Define the asset price without higher order expectations as $P_{t}^{*}=P_{t}-\Delta_{t}$ which from (7) gives $P_{t}^{*}=\bar{E}_{t} P V_{t}-\phi_{t}$. Then it is easy to show that $\operatorname{var}\left(P_{t}\right)<\operatorname{var}\left(P_{t}^{*}\right)$, i.e., that the price is less volatile than it would be if we eliminate the higher order wedge and are left with the two standard asset price components (expected dividends and risk premia). More precisely, $\operatorname{var}\left(P_{t}\right)=\operatorname{var}\left(P_{t}^{*}\right)-\operatorname{var}\left(\Delta_{t}\right)$. This can be seen as follows. Since the asset price $P_{t}$ is in the information set of investors, expectational errors at time $t$ should be orthogonal to the price, so that $\operatorname{cov}\left(P_{t}, \Delta_{t}\right)=0$. Therefore $\operatorname{var}\left(P_{t}^{*}\right)=\operatorname{var}\left(P_{t}-\Delta_{t}\right)=\operatorname{var}\left(P_{t}\right)+\operatorname{var}\left(\Delta_{t}\right)$. It also follows that $\operatorname{cov}\left(P_{t}^{*}, \Delta_{t}\right)=$ $-\operatorname{var}\left(\Delta_{t}\right)$. This negative correlation between the higher order wedge and the sum of the standard asset price components reduces the variance of the price.

The findings that the higher order wedge both reduces asset price volatility and disconnects the price from future dividends are not unrelated. As will be illustrated in the two models below, the equilibrium price gives less weight to future dividends due to the higher order wedge. This both disconnects the price from those future dividends and reduces volatility.

In order to illustrate our general analysis and derive precise results about the impact of the higher order wedge on the equilibrium price, we now turn to two models with specific information and payoff structures. The next section discusses a model with a finitely-lived asset with one payoff. After that we analyze a model with an infinitely-lived asset with continuous payoffs.

\section{A Model With a Finitely-Lived Asset}

As mentioned in Section 2, the case of a finitely-lived asset with only one terminal payoff is well known in finance and has already received some attention in the discussion of higher order expectations in asset pricing. An advantage of this case is that explicit analytical results are feasible. Moreover, we illustrate the main analytical results numerically to show that the quantitative impact of the higher order wedge can be large.

The world starts at date 1 and the asset yields a single dividend of $D_{T}$ at date $T$. Assume that $R=1$. Before trading starts at date 1 all investors receive 
a common signal $\bar{D}=D_{T}+\varepsilon^{d}$, where $\varepsilon^{d} \sim N\left(0, \sigma_{d}^{2}\right)$. Investors also receive a private signal $v^{i}=D_{T}+\varepsilon^{v i}$, with $\varepsilon^{v i} \sim N\left(0, \sigma_{v}^{2}\right)$. The errors in private signals average to zero across investors. It is assumed that investors receive no additional "exogenous" signals subsequent to that, although they will learn from observing the asset price each period. ${ }^{18}$ The asset supplies $X_{1}, . ., X_{T-1}$ are assumed to be i.i.d. $N\left(0, \sigma_{x}^{2}\right)$ variables. ${ }^{19}$

Equation (4) simplifies to:

$$
P_{t}=\bar{E}_{t}^{T-t} D_{T}-\gamma \sigma_{t}^{2} X_{t}
$$

The price depends on the expectation of order $T-t$ of the terminal dividend. The longer the remaining maturity, the higher the order of expectations. The analogue of (7) is given by

$$
P_{t}=\bar{E}_{t} D_{T}-\gamma \sigma_{t}^{2} X_{t}+\Delta_{t}
$$

where the higher order wedge $\Delta_{t}$ is equal to

$$
\Delta_{t}=\bar{E}_{t}^{T-t} D_{T}-\bar{E}_{t} D_{T}
$$

This is the analogue to (5). In this model, the higher order wedge takes a very simple form as the difference between the $T-t$ order expectation and the first order expectation of the terminal dividend.

We now focus on the asset price in period 1. Proposition 1 significantly simplifies in this case because $\bar{V}_{t}=D_{T}$. Moreover, $\Psi_{t}=\theta_{t}$, since $P V_{t}=D_{T}$. Proposition 1 therefore implies that the wedge is equal to

$$
\begin{aligned}
\Delta_{1} & =\pi_{1}\left(\bar{E}_{1} D_{T}-D_{T}\right) \\
& =\left(\theta_{2}+\theta_{2} \theta_{3}+\theta_{2} \theta_{3} \theta_{3}+\ldots+\theta_{2} \theta_{3} \ldots \theta_{T-1}\right)\left(\bar{E}_{1} D_{T}-D_{T}\right)
\end{aligned}
$$

where $\theta_{t}$ is the weight given to the initial private signal in an investor's date- $t$ expectation of the terminal dividend. Since these weights are positive, it follows that $\pi_{1}>0$.

Substitution of the higher order wedge (21) into the equilibrium price (19) for $t=1$ yields

$$
P_{1}=D_{T}+\left(1+\pi_{1}\right)\left(\bar{E}_{1} D_{T}-D_{T}\right)-\gamma \sigma_{t}^{2} X_{t}
$$

\footnotetext{
${ }^{18}$ It is straightforward to extend to the case where investors receive a new private signal each period.

${ }^{19}$ Introducing a non-zero mean asset supply does not qualitatively change any results.
} 
This equation illustrates two implications of higher order expectations discussed in the previous sections. First, the asset price can be written as a function of the first order expectation of future dividends. This also extends the finding of He and Wang (1995) to the relevant dynamic higher order expectations. Second, since $\pi_{1}>0$ equation (22) shows that higher order expectations give more weight to the expectational error about the terminal dividend. As explained in Section 5, this is due to the perfect correlation of expectational errors about future dividends with expectational errors about average private signals, which in this case are both $\bar{E}_{1} D_{T}-D_{T}$. The price will therefore be more disconnected from the future dividend. We make this finding more precise by explicitly solving for $\bar{E}_{1} D_{T}$ from a signal extraction problem. In the Appendix we show that this leads to the following Proposition.

Proposition 5 In the finitely-lived asset case, the equilibrium period 1 asset price depends on the supply shock, the terminal dividend and the common prior $\bar{D}$. Higher order expectations increase the impact of the supply shock and the common prior, while reducing the impact of the terminal dividend. As a result, the correlation between the terminal dividend and the price is reduced by the higher order wedge.

Leaving details to the Appendix, we will sketch the main steps leading to this Proposition. We first conjecture an equilibrium price

$$
P_{1}=\alpha_{1} D_{T}+\alpha_{2} \bar{D}-\alpha_{3} X_{1} \quad \alpha_{1}>0, \alpha_{2}>0, \alpha_{3}>0 \text {. }
$$

An investor's estimate of the terminal dividend is then based on three sources of information. In addition to the private signal, investors have two public signals: the prior $\bar{D}$ and the 'adjusted' asset price $\left(P_{1}-\alpha_{2} \bar{D}\right) / \alpha_{1}$. Thus, the signal extraction problem leads to a simple form of equation (10):

$$
E_{1}^{i} D_{T}=\theta_{1} v^{i}+\gamma_{1}\left(P_{1}-\alpha_{2} \bar{D}\right) / \alpha_{1}+\gamma_{2} \bar{D}
$$

where $\theta_{1}, \gamma_{1}$, and $\gamma_{2}$ are positive scalars that sum to one. The parameters of the equilibrium price equation can be found by substituting the average of (24) across investors into (22) and equating the resulting equilibrium price equation to the conjectured equation (23). The Appendix shows that, as expected, $\alpha_{1}, \alpha_{2}$ and $\alpha_{3}$ are positive. 
Substituting the equilibrium price (23) back into the average of (24) across investors, we have (using $\theta_{1}+\gamma_{1}+\gamma_{2}=1$ ):

$$
\bar{E}_{1} D_{T}-D_{T}=\gamma_{2}\left(\bar{D}-D_{T}\right)-\gamma_{1} \frac{\alpha_{3}}{\alpha_{1}} X_{1}
$$

Since $\Delta_{1}=\pi_{1}\left(\bar{E}_{1} D_{T}-D_{T}\right)$, the higher order wedge depends positively on errors in the public signals: the error $\bar{D}-D_{T}=\varepsilon^{d}$ of the prior signal $\bar{D}$ and the error $-\left(\alpha_{3} / \alpha_{1}\right) X_{1}$ of the price signal. This illustrates the more general result from Proposition 3 that the higher order wedge depends on average expectational errors about average private signals based on public information.

Because the impact of expectational errors on the equilibrium price are amplified by the higher order wedge, the errors in public signals $\bar{D}-D_{T}$ and $-\left(\alpha_{3} / \alpha_{1}\right) X_{1}$ receive more weight in the price. This implies that the impact of the supply shock $X_{1}$ on the price is amplified, the weight on $D_{T}$ is reduced and the weight on $\bar{D}$ is increased. This can be seen directly by substituting (25) into (22):

$$
P_{1}=\left[1-\gamma_{2}\left(1+\pi_{1}\right)\right] D_{T}+\left(1+\pi_{1}\right) \gamma_{2} \bar{D}-\left[\gamma \sigma_{1}^{2}+\left(1+\pi_{1}\right) \gamma_{1} \frac{\alpha_{3}}{\alpha_{1}}\right] X_{1}
$$

where the role of higher order expectations is captured by the parameter $\pi_{1}>0$. By giving less weight to the future payoff and more weight to supply noise the equilibrium asset price is less correlated with the payoff.

Allen et. al. (2003) also study the role of higher order expectations in this model. They do not explicitly consider the higher order wedge. Instead they compare the equilibrium price $P_{t}$ over time. The idea is that the order of the expectation goes down as we get closer to the terminal date. They find that the farther we are from the terminal date, the larger the coefficient on $\bar{D}$ and the smaller coefficient on $D_{T}$. One needs to be careful here since a change in the equilibrium price function over time is affected by both higher order expectations and a change in the information set over time. Their result exclusively reflects the role of higher order expectations in the case where the information set is constant (no learning). This happens when the supply noise has infinite variance, so that the price is not informative. By focusing on the higher order wedge we can investigate the role of higher order expectations in the general case. We find that higher order expectations indeed give more weight to $\bar{D}$ and less weight to $D_{T}$, while in addition the impact of the supply noise on the equilibrium price is amplified. 
Allen et. al. (2003) also emphasize that more weight is given to public information as a result of higher order expectations. More precisely, we find that as a result of higher order expectations more weight is given to errors in public signals. While this result holds in the current example, we show in the next section that it does not generalize to all types of public information.

Figure 1 illustrates the model's properties for a given set of parameters. The Appendix describes the numerical solution method adopted to simultaneously solve for the equilibrium asset prices in all $T$ periods. We consider a benchmark case with $\sigma_{v}=\sigma_{d}=\sigma_{x}=0.4, \gamma=6$ and $T=8$. In Panel A we show the correlation between $P_{t}$ and $D_{T}$ for $1 \leq t \leq 7$ and compare it with the correlation between $P_{t}^{*}$ (the price without the wedge) and $D_{T}$. It shows that the impact of higher order expectations can be very large. In period 1 the correlation between the terminal dividend and the price is less than 0.1 , while the correlation between the terminal dividend and $P^{*}$ is larger than 0.8. As we move closer to the terminal date, the order of expectations goes down and the difference in these correlations is reduced. One period before the terminal date, at $t=7$, the two correlations are equal since there is no higher order expectation.

We should emphasize that panel A only illustrates that the impact of higher order expectations can be very large for certain parameters. It is possible to pick a different set of parameters where the impact is much smaller. In order to judge the true quantitative impact of higher order expectations it is necessary to estimate or calibrate an asset pricing model with more realistic assumptions about the payoff structure, information structure and preferences.

Panel B decomposes the variance of the price as $t$ increases from 1 to 7 . Since $\operatorname{var}\left(P_{t}\right)=\operatorname{var}\left(P_{t}^{*}\right)+\operatorname{var}\left(\Delta_{t}\right)+2 \operatorname{cov}\left(P_{t}^{*}, \Delta_{t}\right)$, we can decompose the variance of the price into three components. They are each reported in panel B as a share of the variance of the price itself. In period 1 both the variance of $P_{t}^{*}$ and the variance of $\Delta_{t}$ are larger than the variance of $P_{t}$, but these two elements have a significant negative covariance. As time goes on the relative variance of the higher order wedge gradually approaches zero.

Finally, Panel C illustrates Proposition 4. The panel shows how much the higher order wedge reduces the correlation between $P_{1}$ and $D_{T}$ in comparison to the correlation between $P_{1}^{*}$ and $D_{T}$. It shows that the correlation between the price and the terminal dividend is reduced the most for an intermediate quality of private 
signals. The peak is reached at $\sigma_{v}=1.1$, where the difference in correlations is close to one. The correlation between the price and the terminal dividend is unaffected by the higher order wedge when the standard deviation of the noise in private signals goes to zero or infinity.

\section{An Infinitely-lived Asset in Steady State}

In the second model we consider, the asset pays dividends each period and has an infinite life. In contrast to the previous model, the information structure is such that the equilibrium price function is time invariant. This case has the advantage that it fits much better within the tradition of dynamic macroeconomic modeling in which time invariant unconditional moments can be computed and compared to the data. The infinite life of the asset does not deliver a time invariant equilibrium by itself. Two other features delivering this are an infinite past and a continuous information flow of constant quality.

We now describe a very simple version of an infinitely-lived asset model. Even though we keep the information structure quite simple, the model does not lend itself to an analytical solution. We therefore solve for the equilibrium price numerically. Although we find that the model exhibits some important differences in comparison to the finite-life asset model, the key finding that the higher order wedge disconnects the asset price from the present value of future dividends is upheld.

The asset yields a dividend $D_{t}$ at each period $t$. For simplicity we assume that dividends are i.i.d., so that:

$$
D_{t}=\bar{D}+\varepsilon_{t}^{d}
$$

where $\varepsilon_{t}^{d} \sim N\left(0, \sigma_{d}^{2}\right)$. The process of dividends is assumed to be common knowledge so that $\bar{D}$ is a public signal. Moreover, each period investors obtain a private signal about the dividend $T$ periods later:

$$
v_{t}^{i}=D_{t+T}+\varepsilon_{t}^{v i}
$$

with $\varepsilon_{t}^{v i} \sim N\left(0, \sigma_{v}^{2}\right)$. It is again assumed that errors in private signals average to zero across investors. Asset supplies $X_{t}$ are again assumed to be i.i.d. $N\left(0, \sigma_{x}^{2}\right)$ variables. This completes the description of the "exogenous" pieces of informa- 
tion available to investors. Equilibrium prices are again an endogenous source of information, to which we turn below.

As $T$ increases investors get information further in advance but also have a larger number of relevant private signals each period. Since $\mathbf{V}_{t}^{i}$ denotes the private information set at time $t$ that is still valuable at time $t+1$, we have $\mathbf{V}_{t}^{i}=\left\{v_{t-T+2}^{i}, . ., v_{t}^{i}\right\}$ and the average across agents is $\overline{\mathbf{V}}_{t}=\left\{D_{t+2}, . ., D_{t+T}\right\}$ for $T \geq 2 .^{20}$ Since expectations of $P V_{t}$ and $\overline{\mathbf{V}}_{t}$ are a time-invariant function of the information set in this model, it follows from Proposition 1 that the vector $\boldsymbol{\Pi}=\frac{1}{R^{2}}(I-\Psi)^{-1} \boldsymbol{\theta}$ is time invariant as well. We will write it as $\boldsymbol{\Pi}^{\prime}=\left\{\pi_{1}, . ., \pi_{T-1}\right\}$. Proposition 1 then implies

$$
\Delta_{t}=\sum_{s=2}^{T} \pi_{s-1}\left(\bar{E}_{t} D_{t+s}-D_{t+s}\right)
$$

Numerical results show that all the elements of $\boldsymbol{\Pi}$ are positive. It is therefore again the case that the higher order wedge depends positively on expectational errors about future dividends and therefore magnifies the impact of expectational errors on the equilibrium price. This will again lower the correlation between the asset price and future dividends.

As in the previous example, expectational errors of future dividends depend on two types of public signal errors, associated with price signals and the signal $\bar{D}$. To compute the equilibrium price, we use the method of undetermined coefficients, and assume the following equation (see the Appendix for a description of the solution method):

$$
P_{t}=\sum_{s=1}^{T} a_{s} D_{t+s}+a_{T+1} \bar{D}-\sum_{s=1}^{T} b_{s} X_{t-s+1} \quad a_{s}>0, b_{s}>0 .
$$

where $\sum_{s=1}^{T+1} a_{s}=1 /(R-1)$. The price is now a signal of a positive weighted average of future dividends. The error in the price signal depends negatively on a weighted average of current and past supply shocks. Even though supply shocks are not persistent, they have a persistent effect on the asset price since past equilibrium prices (which depend on past supply shocks) are informative about

\footnotetext{
${ }^{20}$ When $T=1$ private signals today are no longer in the information set tomorrow since tomorrow's dividend is observed tomorrow. In that case higher order expectations collapse to first order expectations.
} 
future dividends. ${ }^{21}$

Numerical analysis shows that it remains the case, as in the previous model, that the higher order wedge amplifies the contribution of supply shocks to price volatility, while reducing the contribution of future dividends to price volatility. However, it is no longer the case that the higher order wedge reduces the weight on all future dividends and amplifies coefficients on all current and past supply shocks. The reason for this is that some public signals raise the expectation of the present value of future dividends while lowering the expectation of average private signals. To the extent that an error in a public signal raises $\bar{E}_{t} P V_{t}-P V_{t}$ but lowers $\boldsymbol{\Pi}_{t}^{\prime}\left(\bar{E}_{t} \overline{\mathbf{V}}_{t}-\overline{\mathbf{V}}_{t}\right)$, the higher order wedge dampens the impact of the error in the public signal on the equilibrium price. It also explains why the finding by Allen, Morris and Shin (2003) that higher order expectations give more weight to public signals does not easily generalize to all public signals.

Consider for example the case of $T=2$, where both $P_{t}$ and $P_{t-1}$ are part of the public information set. It can be shown that a higher $P_{t-1}$ raises the expectation at time $t$ of the present value of future dividends, but lowers the expectation of the average private signal $\overline{\mathbf{V}}_{t}=D_{t+2} \cdot{ }^{22}$ This leads to a reduced impact of $X_{t-1}$ on $P_{t}$, because $P_{t-1}$ is influenced by $X_{t-1}$. On the other hand, since a higher $P_{t}$ raises expectations of both the present value of future dividends and the average private signal, the impact of $X_{t}$ on the price is amplified. Numerically the overall contribution of supply shocks is still amplified, because the smaller weight on $X_{t-1}$ is dominated by the larger weight on $X_{t}{ }^{23}$

In order to provide an illustration, Figure 2 shows some results for the pa-

\footnotetext{
${ }^{21}$ Supply shocks at $t-T$ and earlier are common knowledge at date $t$ since they can be extracted from equilibrium prices at $t-T$ and earlier.

${ }^{22}$ Since it depends positively on the dividend at $t+1$, a higher $P_{t-1}$ raises the expected dividend at $t+1$. However, $P_{t}$ depends positively on dividends at both $t+1$ and $t+2$. Thus, for a given price $P_{t}$, the expectation of the dividend at $t+2$ is lower the higher the expectation of the dividend at $t+1$.

${ }^{23}$ For the case of $T=2$ we also find that the weight on $D_{t+1}$ is amplified, while the weight on $D_{t+2}$ is reduced. The increased weight on $D_{t+1}$ has a similar explanation as the reduced weight on $X_{t-1}$. To see this it is useful to add the corresponding time index to the prior of a future dividend, e.g., $\bar{D}_{t+1}$ is the prior $D_{t+1}$. Similar to $P_{t-1}$ this public signal raises the expectation of the present value of dividends while lowering the expectation of the average private signal. This leads to a reduced impact on the price of the error $\bar{D}_{t+1}-D_{t+1}$ in the public signal, and therefore a higher weight of $D_{t+1}$.
} 
rameterization $\sigma_{v}=\sigma_{d}=\sigma_{x}=0.4, R=1.02, \gamma=2$. In panels $\mathrm{A}$ and $\mathrm{B}$ the parameter $T$ is varied from 2 to 50 . We should warn not to compare those panels to the corresponding ones from the previous example. In Figure 1 we report how moments vary over time for a given parameterization, while in Figure 2 we report time-independent moments for different parameters $T$ that affect the information structure. Panel A again shows the drop in the correlation between the price and the present value of future dividends as a result of the higher order wedge. The change rises for larger values of $T$. When $T$ is small, the information about most future dividends is public (in the form of the prior $\bar{D}$ ), so that higher order expectations have little impact. The example shows that for $T=50$ the impact of higher order expectations is substantial, reducing the correlation between the present discounted value of dividends and the price from 0.82 to 0.29 .

Consistent with Panel A, Panel B shows that the variance of the higher order wedge rises relative to the variance of the price when $T$ increases. The same is the case for $P^{*}$. When $T=50$ the variance of the higher order wedge is larger than the variance of $P$, while the variance of $P^{*}$ is more than twice the variance of $P$. The remaining factor contributing to the variance of the price is again a large negative covariance between the higher order wedge and $P^{*}$.

Panel C illustrates Proposition 4 for $T=30$. As in the previous example we see that the impact of the higher order wedge on the correlation between the price and the present value of dividends is maximized for an intermediate level of the quality of private information. The reduction in the correlation is largest (0.51) for $\sigma_{v}=0.7$. The impact on the correlation vanishes to zero when either $\sigma_{v} \rightarrow \infty$ or $\sigma_{v} \rightarrow 0$.

\section{Conclusion}

This paper has analyzed the role of higher order expectations for asset pricing. We have shown that higher order expectations generally differ from first order expectations, and that this difference can have strong implications for the equilibrium asset price. The paper has devoted significant attention towards understanding what determines this new asset pricing determinant, which we called the "higher order wedge," and how it affects the equilibrium price. A key result is that it weakens the relationship between the price and future dividends. 
While our analysis assumes full rationality of investors, the recent literature in behavioral finance implies that expectational errors could be caused by deviations from rationality, such as overconfidence or changing market mood. We conjecture that the insights from our general analysis also apply when expectational errors are caused by factors different from noisy public signals. In particular, the impact of these errors would be amplified by higher order expectations. Combining the dimension of market psychology with our analysis of higher order expectations would bring us close to Keynes' reasoning on asset prices and closer to understanding asset price movements.

Another natural direction for future research is to quantify the importance of the higher order wedge as an asset pricing determinant. While we have shown that it can be quantitatively very large, its magnitude needs to be evaluated in the context of a somewhat more realistic setup that is calibrated to actual data. In particular, we have maintained the standard assumption in noisy rational expectations models of constant absolute risk aversion. While this simplifies the solution significantly, a more realistic constant rate of relative risk aversion needs to be adopted when confronting the model to the data. More realistic assumptions about the process of dividends and the information structure would need to be considered as well. 


\section{Appendix}

\section{A Proof of Proposition 1}

Using Assumption 2, the investor's expectation of (13) is:

$$
E_{t}^{i}\left(\bar{E}_{t+1} P V_{t+1}-E_{t+1}^{i} P V_{t+1}\right)=\boldsymbol{\theta}_{t+1}^{\prime}\left(E_{t}^{i}\left(\overline{\mathbf{V}}_{t}\right)-\mathbf{V}_{t}^{i}\right)
$$

so that average expectation at time $t$ of the expected average expectational error at $t+1$ is:

$$
\bar{E}_{t}\left(\bar{E}_{t+1} P V_{t+1}-P V_{t+1}\right)=\boldsymbol{\theta}_{t+1}^{\prime}\left(\bar{E}_{t} \overline{\mathbf{V}}_{t}-\overline{\mathbf{V}}_{t}\right)
$$

The other terms in (8) involve higher order expectations of future expectational errors. Consider the deviation at $t+s: \bar{E}_{t}^{s}\left(\bar{E}_{t+s} P V_{t+s}-P V_{t+s}\right)$. It can be rewritten as $\bar{E}_{t}^{s-1} \bar{E}_{t+s-1}\left(\bar{E}_{t+s} P V_{t+s}-P V_{t+s}\right)$. Using (32) at time $t+s-1$ we can write:

$$
\bar{E}_{t}^{s}\left(\bar{E}_{t+s} P V_{t+s}-P V_{t+s}\right)=\boldsymbol{\theta}_{t+s}^{\prime} \bar{E}_{t}^{s-1}\left(\bar{E}_{t+s-1} \overline{\mathbf{V}}_{t+s-1}-\overline{\mathbf{V}}_{t+s-1}\right)
$$

This implies that investors at time $t$ need to compute higher order expectations of information available to investors at time $t+s-1$.

Using (11) and following the same reasoning as to get (32), it then follows that

$$
\bar{E}_{t}\left(\bar{E}_{t+1} \overline{\mathbf{V}}_{t+1}-\overline{\mathbf{V}}_{t+1}\right)=\mathbf{\Psi}_{t+1}^{\prime}\left(\bar{E}_{t} \overline{\mathbf{V}}_{t}-\overline{\mathbf{V}}_{t}\right)
$$

Similarly $\bar{E}_{t+s-2}\left(\bar{E}_{t+s-1} \overline{\mathbf{V}}_{t+s-1}-\overline{\mathbf{V}}_{t+s-1}\right)=\mathbf{\Psi}_{t+s-1}^{\prime}\left(\bar{E}_{t+s-2} \overline{\mathbf{V}}_{t+s-2}-\overline{\mathbf{V}}_{t+s-2}\right)$. This can be substituted into (33) and we can work backwards using (11) to get

$$
\bar{E}_{t}^{s}\left(\bar{E}_{t+s} P V_{t+s}-P V_{t+s}\right)=\boldsymbol{\theta}_{t+s-1}^{\prime} \Psi_{t+s-1}^{\prime} \ldots \Psi_{t+1}^{\prime}\left(\bar{E}_{t} \overline{\mathbf{V}}_{t}-\overline{\mathbf{V}}_{t}\right)
$$

Doing this for all $s$ and adding it to (32) gives $\Delta_{t}$ in Proposition 1.

\section{B Proof of Proposition 4}

Define $\mathbf{m}_{t}=\operatorname{cov}\left(\overline{\mathbf{W}}_{t}, P V_{t} \mid \mathbf{Z}_{t}\right), \boldsymbol{\Omega}_{t}=\operatorname{var}\left(\overline{\mathbf{W}}_{t} \mid \mathbf{Z}_{t}\right)$ and $\boldsymbol{\Sigma}_{t}=\operatorname{var}\left(\mathbf{W}_{t}^{i}-\overline{\mathbf{W}}_{t}\right)$. Let $\mathbf{A}_{t}$ and $\mathbf{B}_{t}$ be matrices of zeros and ones such that $\overline{\mathbf{V}}_{t}=\mathbf{A}_{t} \overline{\mathbf{W}}_{t}$ and $\overline{\mathbf{V}}_{t-1}=\mathbf{B}_{t} \overline{\mathbf{W}}_{t}$. It then follows from Proposition 1 and (15) that

$$
\Delta_{t}=\boldsymbol{\Pi}_{t}^{\prime} \mathbf{A}_{t} \boldsymbol{\Sigma}_{t}\left(\boldsymbol{\Omega}_{t}+\boldsymbol{\Sigma}_{t}\right)^{-1}\left(E_{t}\left(\overline{\mathbf{W}}_{t} \mid \mathbf{Z}_{t}\right)-\overline{\mathbf{W}}_{t}\right)
$$


Therefore

$$
\operatorname{var}\left(\Delta_{t}\right)=\boldsymbol{\Pi}_{t}^{\prime} \mathbf{A}_{t} \boldsymbol{\Sigma}_{t}\left(\boldsymbol{\Omega}_{t}+\boldsymbol{\Sigma}_{t}\right)^{-1} \boldsymbol{\Omega}_{t}\left(\boldsymbol{\Omega}_{t}+\boldsymbol{\Sigma}_{t}\right)^{-1} \boldsymbol{\Sigma}_{t} \mathbf{A}_{t}^{\prime} \boldsymbol{\Pi}_{t}
$$

The vector $\boldsymbol{\Pi}_{t}$ depends on $\boldsymbol{\theta}_{t+s}$ and $\boldsymbol{\Psi}_{t+s}, s=1,2, \ldots$ From the projection theorem $\partial E_{t+s}^{i} P V_{t+s} / \partial \mathbf{W}_{t+s}^{i}=\mathbf{m}_{t+s}^{\prime}\left(\boldsymbol{\Omega}_{t+s}+\boldsymbol{\Sigma}_{t+s}\right)^{-1}$. Thus $\boldsymbol{\theta}_{t+s}=\mathbf{B}_{t+s}\left(\boldsymbol{\Omega}_{t+s}+\right.$ $\left.\boldsymbol{\Sigma}_{t+s}\right)^{-1} \mathbf{m}_{t+s}$. Similarly, from the projection theorem $\partial E_{t+s}^{i} \overline{\mathbf{W}}_{t+s} / \partial \mathbf{W}_{t+s}^{i}=\boldsymbol{\Omega}_{t+s}\left(\boldsymbol{\Omega}_{t+s}+\right.$ $\left.\boldsymbol{\Sigma}_{t+s}\right)^{-1}$. Therefore $\boldsymbol{\Psi}_{t+s}=\mathbf{B}_{t+s}\left(\boldsymbol{\Omega}_{t+s}+\boldsymbol{\Sigma}_{t+s}\right)^{-1} \boldsymbol{\Omega}_{t+s} \mathbf{A}_{t+s}^{\prime}$.

First consider the case where the idiosyncratic noise in private signals approaches zero, so that $\Sigma_{t} \rightarrow 0$ for all $t$. All other matrices are unaffected. It follows that $\boldsymbol{\theta}_{t+s} \rightarrow \mathbf{B}_{t+s} \boldsymbol{\Omega}_{t+s}^{-1} \mathbf{m}_{t+s}$ and $\boldsymbol{\Psi}_{t+s} \rightarrow \mathbf{B}_{t+s} \mathbf{A}_{t+s}^{\prime}$, so that $\boldsymbol{\Pi}_{t}$ approaches some vector of finite values. From (36) it immediately follows that $\operatorname{var}\left(\Delta_{t}\right) \rightarrow 0$ since the expression $\mathbf{A} \boldsymbol{\Sigma}_{t}\left(\boldsymbol{\Omega}_{t}+\boldsymbol{\Sigma}_{t}\right)^{-1} \boldsymbol{\Omega}_{t}\left(\boldsymbol{\Omega}_{t}+\boldsymbol{\Sigma}_{t}\right)^{-1} \boldsymbol{\Sigma}_{t} \mathbf{A}^{\prime}$ approaches zero.

Next consider the case where the idiosyncratic errors in private signals become infinitely noisy. More precisely, for all $t \boldsymbol{\Sigma}_{t} \rightarrow k \bar{\Sigma}_{t}$ with $k \rightarrow \infty$ and $\overline{\boldsymbol{\Sigma}}_{t}$ a matrix with finite values. It follows that $\boldsymbol{\theta}_{t+s} \rightarrow 0$ and $\boldsymbol{\Psi}_{t+s} \rightarrow 0$, so that $\boldsymbol{\Pi}_{t} \rightarrow 0$. The expression $\mathbf{A}_{t} \boldsymbol{\Sigma}_{t}\left(\boldsymbol{\Omega}_{t}+\boldsymbol{\Sigma}_{t}\right)^{-1} \boldsymbol{\Omega}_{t}\left(\boldsymbol{\Omega}_{t}+\boldsymbol{\Sigma}_{t}\right)^{-1} \boldsymbol{\Sigma}_{t} \mathbf{A}_{t}^{\prime}$ in (36) approaches the matrix $\mathbf{A}_{t} \boldsymbol{\Omega}_{t} \mathbf{A}_{t}^{\prime}$, which has finite values. Therefore $\operatorname{var}\left(\Delta_{t}\right) \rightarrow 0$. This completes the proof of Proposition 4 .

\section{Finitely-lived Asset}

In this section we solve the model of Section 6 and prove Proposition 5.

\section{C.1 Model Solution}

First, we can use (2) to write the asset price at each period:

$$
\begin{array}{rlr}
P_{t} & =\bar{E}_{t}\left(P_{t+1}\right)-\gamma \sigma_{t}^{2} X_{t} & t<T-1 \\
P_{T-1} & =\bar{E}_{T-1}\left(D_{T}\right)-\gamma \sigma_{T-1}^{2} X_{T-1} &
\end{array}
$$

where $\sigma_{t}^{2}=\operatorname{var}_{t}\left(P_{t+1}\right)$. We now conjecture equilibrium prices for $1 \leq t<T$ :

$$
\begin{aligned}
P_{1}= & a_{1} D_{T}+b_{1} \bar{D}+c_{11} X_{1} \\
P_{2}= & a_{2} D_{T}+b_{2} \bar{D}+c_{21} X_{1}+c_{22} X_{2} \\
& \ldots \\
P_{T-1}= & a_{T-1} D_{T}+b_{T-1} \bar{D}+c_{T-1,1} X_{1}+c_{T-1,2} X_{2}+\ldots+c_{T-1, T-1} X_{T-1}
\end{aligned}
$$


Besides the prior $\bar{D}$, the information available to investors at time $t$ consists of prices and the private signal. It is useful to define the adjusted price signals as $\widetilde{P}_{t} \equiv P_{t}-b_{t} \bar{D}$ (as a signal of $a_{t} D_{T}$ ). Define the vector of unobservables as $K_{t}=$ $\left(D_{T}, X_{1}, X_{2}, \ldots, X_{t}\right)^{\prime}$. Based on prior information this has a normal distribution with mean $\bar{K}=(\bar{D}, 0, \ldots, 0)^{\prime}$ and variance

$$
\Sigma_{K K, t}=\left[\begin{array}{cccc}
\sigma_{d}^{2} & 0 & \ldots & 0 \\
0 & \sigma_{x}^{2} & \ldots & 0 \\
\vdots & \vdots & \ddots & \vdots \\
0 & 0 & \ldots & \sigma_{x}^{2}
\end{array}\right]
$$

Now consider updating the expectation and variance of $K_{t}$ based on the vector of observables $Y_{t}^{i}=\left(\widetilde{P}_{1}, \widetilde{P}_{2}, \ldots, \widetilde{P}_{t}, v^{i}\right)^{\prime}$. Using (39) we can write:

$$
Y_{t}^{i}=C_{t} K_{t}+s_{t}^{i}
$$

where $s_{t}^{i}=\left(0, \ldots, 0, \varepsilon^{v i}\right)^{\prime}$ and

$$
C_{t}=\left[\begin{array}{ccccc}
a_{1} & c_{11} & 0 & \ldots & 0 \\
a_{2} & c_{21} & c_{22} & \ldots & 0 \\
\vdots & \vdots & \vdots & \ddots & \vdots \\
a_{t} & c_{t 1} & c_{t 2} & \ldots & c_{t t} \\
1 & 0 & 0 & \ldots & 0
\end{array}\right] \quad \Sigma_{s s, t}=\operatorname{var}\left(s_{t}^{i}\right)=\left[\begin{array}{cccc}
0 & 0 & \ldots & 0 \\
0 & 0 & \ldots & 0 \\
\vdots & \vdots & \ddots & \vdots \\
0 & 0 & \ldots & \sigma_{v}^{2}
\end{array}\right]
$$

Applying the projection theorem, we get:

$$
\begin{aligned}
E_{t}^{i} K_{t} & =\bar{K}+\Sigma_{K K, t} C_{t}^{\prime}\left(C_{t} \Sigma_{K K, t} C_{t}^{\prime}+\Sigma_{s s, t}\right)^{-1}\left(Y_{t}^{i}-C_{t} \bar{K}\right) \\
& \equiv\left(I-M_{t} C_{t}\right) \bar{K}+M_{t} Y_{t}^{i} \\
\operatorname{var}_{t}\left(K_{t}\right) & =\Sigma_{K K, t}-\Sigma_{K K, t} C_{t}^{\prime}\left(C_{t} \Sigma_{K K, t} C_{t}^{\prime}+\Sigma_{s s, t}\right)^{-1} C_{t} \Sigma_{K K, t}^{\prime}
\end{aligned}
$$

We can now consider $P_{t+1}$ and take its expectation. The conjecture for $P_{t+1}$ is:

$$
P_{t+1}=a_{t+1} D_{T}+b_{t+1} \bar{D}+c_{t+1,1} X_{1}+c_{t+1,2} X_{2}+\ldots+c_{t+1, t+1} X_{t+1}
$$

which can be rewritten as:

$$
P_{t+1}=b_{t+1} \bar{D}+A_{t+1}^{\prime} K_{t}+c_{t+1, t+1} X_{t+1}
$$


where $A_{t+1}^{\prime}=\left(a_{t+1}, c_{t+1,1}, \ldots, c_{t+1, t}\right)$. Using (41), we can write:

$$
E_{t}^{i}\left(P_{t+1}\right)=\bar{b}_{t+1} \bar{D}+A_{t+1}^{\prime} M_{t} Y_{t}^{i}
$$

where $\bar{b}_{t+1}$ is $b_{t+1}$ plus $A_{t+1}^{\prime}$ times the first column of $\left(I-M_{t} C_{t}\right)$. Aggregating over investors, using (40) and the fact that $s_{t}^{i}$ averages to zero, we have:

$$
\bar{E}_{t}\left(P_{t+1}\right)=\bar{b}_{t+1} \bar{D}+A_{t+1}^{\prime} M_{t} C_{t} K_{t}
$$

The variance is given by:

$$
\sigma_{t}^{2}=\operatorname{var}_{t}\left(P_{t+1}\right)=A_{t+1}^{\prime} \operatorname{var}_{t}\left(K_{t}\right) A_{t+1}+c_{t+1, t+1}^{2} \sigma_{x}^{2}
$$

The expectation of $P_{t+1}$ can be substituted into (37) so that for $t<T-1$ :

$$
\begin{aligned}
P_{t}= & \bar{b}_{t+1} \bar{D}+A_{t+1}^{\prime} M_{t} C_{t} K_{t}-\gamma \sigma_{t}^{2} X_{t} \equiv \\
& \widetilde{a}_{t} D_{T}+\widetilde{b}_{t} \bar{D}+\widetilde{c}_{t, 1} X_{1}+\widetilde{c}_{t, 2} X_{2}+\ldots+\widetilde{c}_{t, t} X_{t}
\end{aligned}
$$

This equation has the same form as the conjectured equation (39) for $1 \leq t<T-1$. For $t=T-1$ we can derive a similar equation from $(41)$, using that $\bar{E}_{T-1}\left(D_{T}\right)$ is equal to the first element of $E_{T-1}^{i} K_{T-1}$. This again yields an equilibrium price equation that has the same form as the conjectured price equation. All that remains is to equate the coefficients of the equilibrium price equations to the coefficients of the conjectured price equations. This involves solving a fixed point problem for a set of non-linear equations, which is done numerically.

\section{C.2 Proposition 5}

Section 6 focuses on the price at $t=1$. In this case we have $Y_{1}^{i}=\left(\widetilde{P}_{1}, v^{i}\right)^{\prime}$ and $K_{1}=\left(D_{T}, X_{1}\right)^{\prime}$ with the matrices:

$$
C_{1}=\left[\begin{array}{cc}
a_{1} & c_{11} \\
1 & 0
\end{array}\right] \quad \Sigma_{K K, 1}=\left[\begin{array}{cc}
\sigma_{d}^{2} & 0 \\
0 & \sigma_{x}^{2}
\end{array}\right] \quad \Sigma_{s s, 1}=\left[\begin{array}{cc}
0 & 0 \\
0 & \sigma_{v}^{2}
\end{array}\right]
$$

This implies that:

$$
M_{1}=\frac{1}{S}\left[\begin{array}{ll}
m_{11} & m_{12} \\
m_{21} & m_{22}
\end{array}\right]
$$


with $m_{11}=a_{1} \sigma_{d}^{2} \sigma_{v}^{2}, m_{12}=c_{11}^{2} \sigma_{x}^{2} \sigma_{d}^{2}, m_{21}=c_{11} \sigma_{x}^{2}\left(\sigma_{d}^{2}+\sigma_{v}^{2}\right), m_{22}=-a_{1} c_{11} \sigma_{x}^{2} \sigma_{d}^{2}$, and $S=a_{1}^{2} \sigma_{d}^{2} \sigma_{v}^{2}+c_{11}^{2} \sigma_{x}^{2} \sigma_{d}^{2}+c_{11}^{2} \sigma_{x}^{2} \sigma_{v}^{2}$.

Using (41), we find:

$$
\bar{E}_{1} D_{T}=\left(1-\frac{a_{1} m_{11}+m_{12}}{S}\right) \bar{D}+\frac{1}{S}\left(m_{11} \widetilde{P}_{1}+m_{12} D_{T}\right)
$$

which can be rewritten as:

$$
\bar{E}_{1} D_{T}=\frac{\frac{1}{\sigma_{d}^{2}} \bar{D}+\frac{1}{\sigma_{v}^{2}} D_{T}+\frac{a_{1}}{c_{11}^{2}} \frac{1}{\sigma_{x}^{2}} \widetilde{P}_{1}}{\frac{1}{\sigma_{d}^{2}}+\frac{1}{\sigma_{v}^{2}}+\left(\frac{a_{1}}{c_{11}}\right)^{2} \frac{1}{\sigma_{x}^{2}}}
$$

which leads to (24), with $\theta_{1}, \gamma_{1}$ and $\gamma_{2}$ the coefficients on respectively $\bar{D}, D_{T}$ and $\tilde{P}_{1} / a_{1}$. The expectation in (44) can be substituted into (22):

$$
P_{1}=\left(1+\pi_{1}\right)\left[\theta_{1} D_{T}+\frac{\gamma_{1}}{a_{1}}\left(P_{1}-b_{1} \bar{D}\right)+\gamma_{2} \bar{D}\right]-\pi_{1} D_{T}-\gamma \sigma_{1}^{2} X_{t}
$$

so that:

$$
\left[1-\left(1+\pi_{1}\right) \frac{\gamma_{1}}{a_{1}}\right] P_{1}=\left[\left(1+\pi_{1}\right) \theta_{1}-\pi_{1}\right] D_{T}+\left(1+\pi_{1}\right)\left[\gamma_{2}-\frac{\gamma_{1} b_{1}}{a_{1}}\right] \bar{D}-\gamma \sigma_{1}^{2} X_{t}
$$

To determine the sign of $a_{1}, b_{1}$, and $c_{11}$, we use the fact that $\pi_{1}<\frac{\theta_{1}}{1-\theta_{1}}$. This inequality holds for the following reason. Let $\mathbf{Z}_{t}=\left(\bar{D}, \widetilde{P}_{1}, \widetilde{P}_{2}, \ldots, \widetilde{P}_{t}\right)^{\prime}$ be the set of public information. Since this set of public information only expands over time it follows that $\operatorname{var}\left(D_{T} \mid \mathbf{Z}_{t}\right) \leq \operatorname{var}\left(D_{T} \mid \mathbf{Z}_{1}\right)$. Using the projection theorem, it follows that when adding the private signal $v^{i}$ to this public information set, the coefficient on $v^{i}$ in the expectation of $D_{T}$ is equal to $\theta_{t}=\operatorname{var}\left(D_{T} \mid \mathbf{Z}_{t}\right) /\left(\operatorname{var}\left(D_{T} \mid \mathbf{Z}_{t}\right)+\sigma_{v}^{2}\right)$. It follows that $\theta_{t} \leq \theta_{1}$ and therefore $\pi_{1} \leq \theta_{1}+\theta_{1}^{2}+\ldots+\theta_{1}^{T-2}<\frac{\theta_{1}}{1-\theta_{1}}$ since the latter term is the limit for the infinite series $\theta_{1}^{n}$.

Using the conjectured price equation (23), the notation $\alpha_{1} \equiv a_{1}, \alpha_{2} \equiv b_{1}$, $\alpha_{3} \equiv c_{11}$, and $\pi_{1}<\frac{\theta_{1}}{1-\theta_{1}}$, from (45) we have:

$$
\begin{aligned}
& \alpha_{1}=\frac{\left(1+\pi_{1}\right) \theta_{1}-\pi_{1}}{1-\left(1+\pi_{1}\right) \frac{\gamma 1}{\alpha_{1}}}>0 \rightarrow \alpha_{1}=\left(1+\pi_{1}\right)\left(\theta_{1}+\gamma_{1}\right)-\pi_{1}>0 \\
& \alpha_{2}=\frac{\left(1+\pi_{1}\right)\left(\gamma_{2}-\frac{\gamma_{1} \alpha_{2}}{\alpha_{1}}\right)}{1-\left(1+\pi_{1}\right) \frac{\gamma_{1}}{\alpha_{1}}}>0 \rightarrow \alpha_{2}=\gamma_{2}\left(1+\pi_{1}\right)>0 \\
& \alpha_{3}=\frac{\gamma \sigma_{1}^{2}}{1-(1+\pi 1) \frac{\gamma 1}{\alpha 1}}=\left(1+\frac{\left(1+\pi_{1}\right) \gamma_{1}}{\left(1+\pi_{1}\right) \theta_{1}-\pi_{1}}\right) \gamma \sigma_{1}^{2}>0
\end{aligned}
$$

It can be easily seen that $\pi_{1}>0$ lowers $\alpha_{1}$, while raising $\alpha_{2}$ and $\alpha_{3}$. The rest of the proof of Proposition 5 follows from the discussion in Section 5. 


\section{Infinitely-Lived Asset}

Without going into great detail we provide a brief overview of the solution method for the model in section 7 of the paper. The starting point for the solution is equation (2) and the conjectured price equation (23):

$$
P_{t}=\sum_{s=1}^{T} a_{s} D_{t+s}+a_{T+1} \bar{D}-\sum_{s=1}^{T} b_{s} X_{t-s+1} \quad a_{s}>0, b_{s}>0 .
$$

The vector of unknowns in this case is $K_{t}=\left(D_{t+1}, . ., D_{t+T}, X_{t-T+1}, \ldots, X_{t}\right)^{\prime}$. At time $t$ investors know supply shocks at $t-T$ and earlier from the equilibrium prices at $t-T$ and earlier. Prices from $t-T+1$ to $t$ are informative about the vector $K_{t}$ of unknowns. It is useful to define adjusted prices as $\tilde{P}_{t-s}=P_{t-s}-a_{T+1} \bar{D}-$ $\sum_{k=0}^{s-1} b_{T-s+1+k} X_{t-T-k}$, which at time $t$ are observables for $s \geq 0$.

As in the case of the finitely-lived asset case, we update the distribution of $K_{t}$ based on prior information with a vector of observables, which now is $Y_{t}^{i}=$ $\left(\tilde{P}_{t-T+1}, . ., \tilde{P}_{t}, v_{t-T+1}^{i}, . ., v_{t}^{i}\right)^{\prime}$. We again use the fact that $Y_{t}^{i}$ can be written as $C K_{t}+$ $s_{t}^{i}$, where now $s_{t}^{i}=\left(0 . .0, \epsilon_{t-T+1}^{v i}, . ., \epsilon_{t}^{v i}\right)^{\prime}$. Applying the projection theorem, and averaging over investors, $\bar{E}_{t} K_{t}=(I-M C) \bar{K}+M C K_{t}$, where $\bar{K}=(\bar{D}, . ., \bar{D}, 0 . .0)^{\prime}$ is the expectation of $K_{t}$ based on prior information and $M$ is the same matrix as in Appendix C. The variance of $K_{t}$ also takes the same form as in Appendix C. Using the conjectured price equation, we can then compute the average expectation of $P_{t+1}+D_{t+1}$ as a function of $\bar{K}$ and $K_{t}$, as well as the variance of $P_{t+1}+D_{t+1}$ (which is $\sigma^{2}$ ). Substituting this into (2) and using the definition of $\phi_{t}$ we obtain the equilibrium period- $t$ price as a function of $\bar{K}$ and $K_{t}$, which therefore takes the same form as the conjectured price equation. All that remains to be done is to equate the conjectured parameters to the equilibrium parameters. This non-linear fixed point problem is solved numerically. 


\section{References}

[1] Admati, Anat R. (1985), "A Noisy Rational Expectations Equilibrium for Multi-Asset Securities Markets," Econometrica 53, 629-657.

[2] Allen, Franklin, Stephen Morris, and Hyun Song Shin (2003), "Beauty Contests, Bubbles and Iterated Expectations in Asset Markets," mimeo.

[3] Amato, Jeffery D., and Hyun Song Shin (2003), "Public and Private information in Monetary Policy Models," BIS Working Paper No. 138.

[4] Bacchetta, Philippe and Eric van Wincoop (2003), "Can Information Heterogeneity Explain the Exchange Rate Determination Puzzle?" NBER WP No. 9498.

[5] Barberis, Nicholas, and Richard Thaler (2003), "A Survey of Behavioral Finance," in George Constantinides, Milton Harris, and Rene Stulz (eds.), Handbook of the Economics of Finance, Elsevier, Amsterdam.

[6] Biais, Bruno, and Peter Bossaerts (1998), "Asset Prices and Trading Volume in a Beauty Contest," Review of Economic Studies 65, 307-340.

[7] Brennan, Michael J., and H. Henry Cao (1997), "International Portfolio Investment Flows," Journal of Finance LII(5), 1851-1880.

[8] Brunnermeier, Markus K. (2001), Asset Pricing under Asymmetric Information, Oxford University Press, (Oxford).

[9] Foster, F. Douglas, and S. Viswanathan (1996), "Strategic Trading When Agents Forecast the Forecasts of Others," Journal of Finance 51, 1437-1478.

[10] He, Hua, and Jiang Wang (1995), "Differential Information and Dynamic Behavior of Stock Trading Volume," Review of Financial Studies 8, 919-972.

[11] Hellwig, Christian (2002), "Public Announcements, Adjustment Delays and the Business Cycle," mimeo, UCLA.

[12] Hirshleifer, David (2001), "Investor Psychology and Asset Pricing," Journal of Finance 56, 1533-1597. 
[13] Keynes, John Maynard (1936), The General Theory of Employment, Interest and Money, MacMillan, London.

[14] Morris, Stephen and Hyun Song Shin (2002), "Social Values of Public Information," American Economic Review 92, 1521-1534.

[15] Singleton, Kenneth J. (1987), "Asset Prices in a Time-Series Model with Disparately Informed, Competitive Traders", in W.A. Barnett, and K.J. Singleton, eds., New Approaches to Monetary Economics, Cambridge University Press (Cambridge, UK), 249-272.

[16] Townsend, Robert M. (1983), "Forecasting the Forecasts of Others," Journal of Political Economy 91, 546-588.

[17] Vives, Xavier (1995), "Short-Term Investment and the Informational Efficiency of the Market," Review of Financial Studies 8, 125-160.

[18] Woodford, Michael (2003), "Imperfect Common Knowledge and the Effects of Monetary Policy," in Ph. Aghion, R. Frydman, J. Stiglitz, and M. Woodford (eds) Knowledge, Information and Expectations in Modern Macroeconomics: In Honor of Edmund S. Phelps, Princeton University Press. 


\section{Figure 1 Numerical Illustration Finitely-Lived Asset*}

Panel A: Correlation between Price and Terminal Dividend

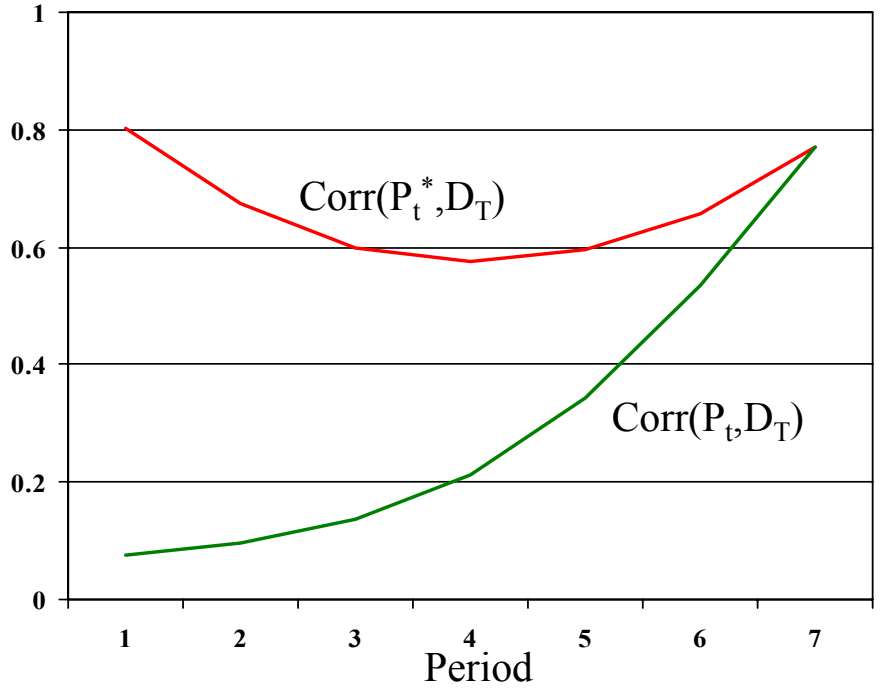

Panel C: Private Signal Noise and Higher Order Wedge

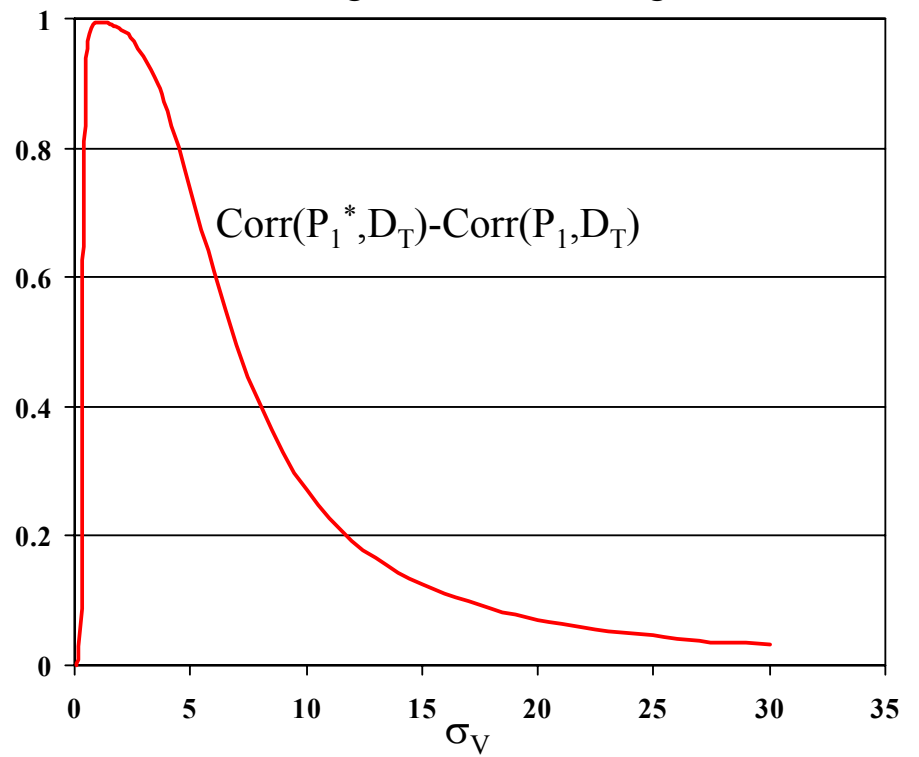

* Benchmark parameters: $\sigma_{\mathrm{d}}=\sigma_{\mathrm{v}}=\sigma_{\mathrm{x}}=0.4 ; \gamma=6$.
Panel B: Variance Decomposition of the Price

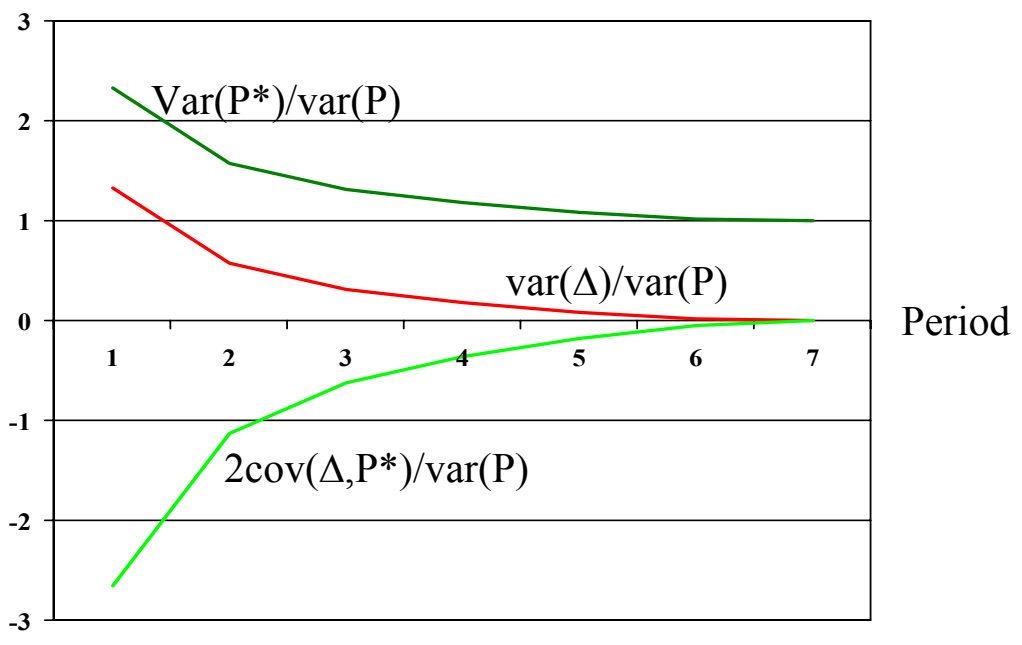




\section{Figure 2 Numerical Illustration Infinitely-Lived Asset*}

Panel A: Correlation between Price and PV

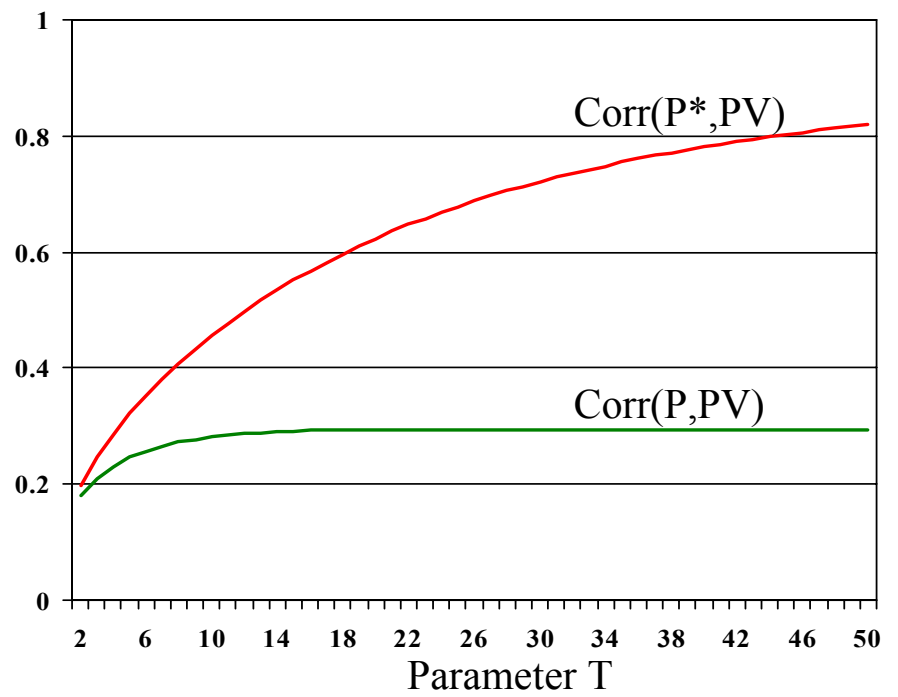

Panel B: Variance Decomposition of the Price

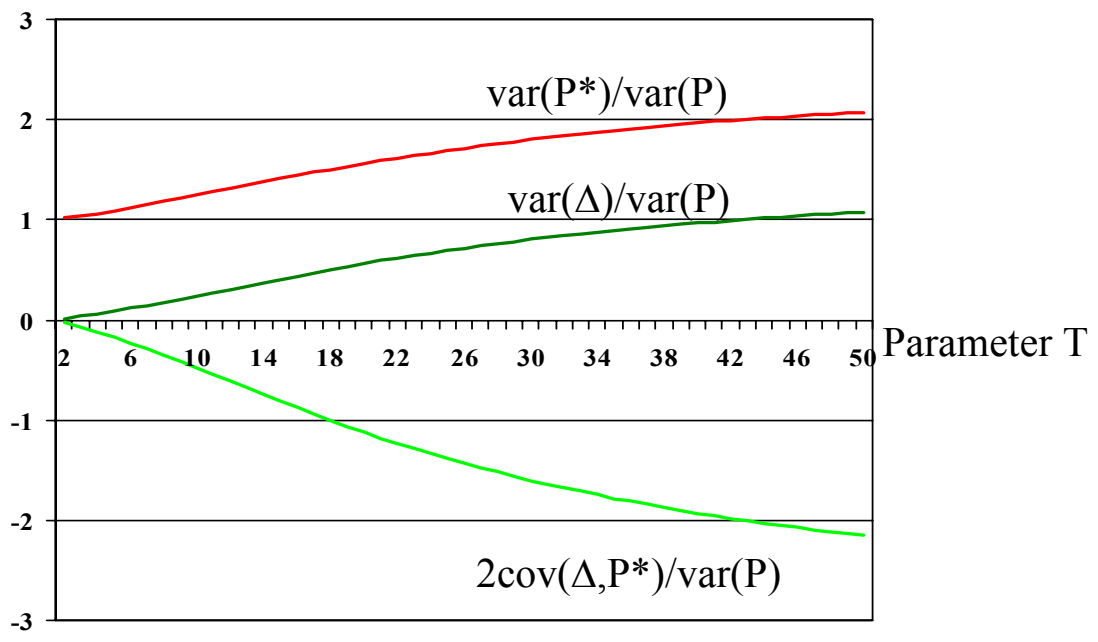

Panel C: Private Signal Noise and Higher Order Wedge

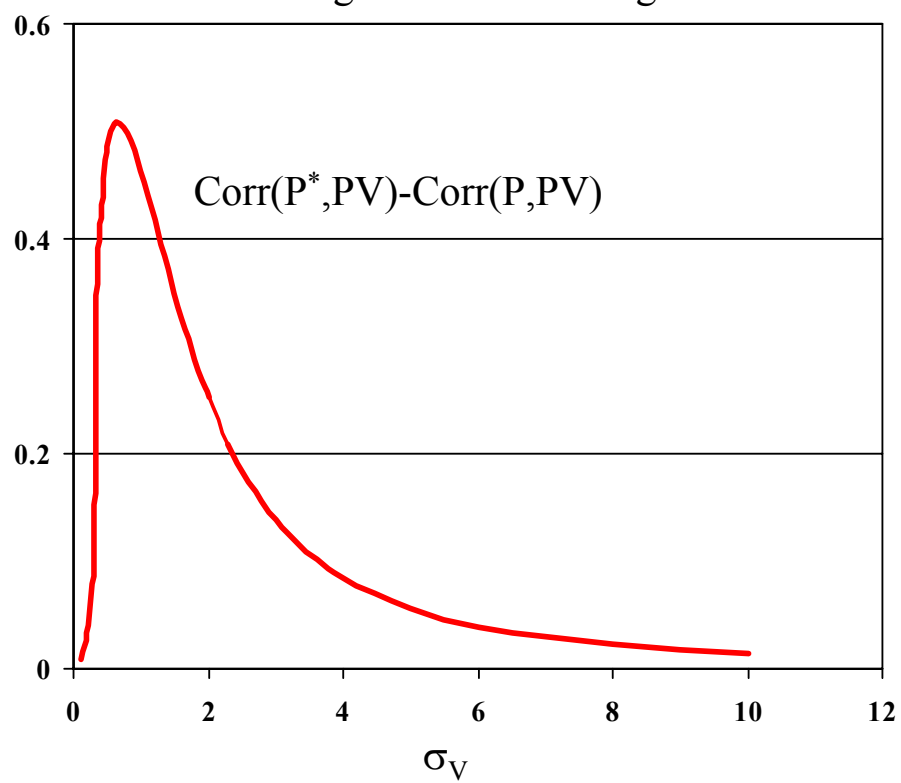

* Benchmark parameters: $\mathrm{T}=30, \sigma_{\mathrm{d}}=\sigma_{\mathrm{v}}=\sigma_{\mathrm{x}}=0.4 ; \mathrm{R}=1.02, \gamma=2$. 